\title{
Erzeugt nicht-diagnostische Information einen Reihenfolge-Effekt im Fall der attributweisen Informationspräsentation?
}
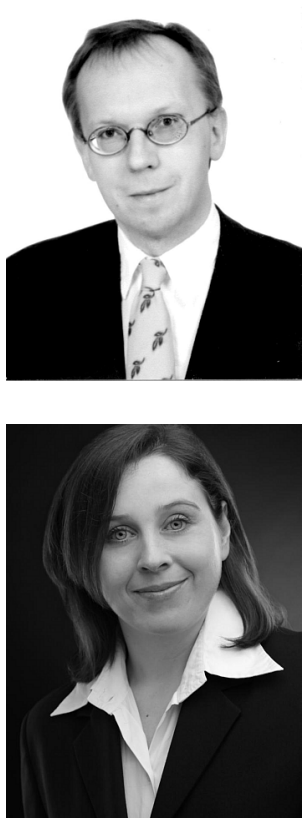

Heribert Gierl und Karin Stiegelmayr

Attributweise Informationspräsentation, Primacy-Effekt, Verkaufsgespräch, Online-Shop

Attribute-based information presentation, Primacy effect, Sales conversation, Online shop

Hier wird die Situation betrachtet, in der Konsumenten zwei Wahlmöglichkeiten haben, zwischen denen sie eine Entscheidung treffen. Dazu erhalten sie Informationen über die Merkmale der Alternativen. Konsumenten müssen die Informationseinheiten zwangsläufig in einer bestimmten Reihenfolge verarbeiten. Diese Reihenfolge wirkt sich auf den Ausgang des Entscheidungsprozesses aus. Das Forschungsgebiet zu Reihenfolge-Effekten ist sehr breit. In diesem Beitrag wird für ein noch kaum erforschtes Teilgebiet untersucht, wie im Fall einer attributweisen Informationspräsentation ein systematischer Reihenfolge-Effekt erzeugt werden kann. Es wird gezeigt, dass die Einbindung von nicht-diagnostischer Information zwischen der Darstellung der Vor- und Nachteile der Wahlmöglichkeiten einen Primacy-Effekt auslöst, d. h. einen Präferenzvorteil für die Option, deren Vorteil am Anfang der Informationspräsentation aufgezeigt wird. Diese Erkenntnis kann bei der Gestaltung des Verkaufsgesprächs oder der Programmierung von Online-Shops genutzt werden, um den Wahlanteil eines bestimmten Produkts gezielt zu erhöhen.

We focus on the situation where consumers have to come to a decision for one of two alternatives. We consider scenarios where the alternatives are described by relative advantages and disadvantages, respectively. Under this condition, consumers process information sequentially. It is well-known that the sequence of information presentation causes order effects on decisions. However, a certain case has rarely been investigated yet: the case of attribute-based information processing. We show that inserting non-diagnostic information into the presentation of advantages and disadvantages causes a primacy effect. If an alternative's advantages are shown first and its disadvantages are shown in the final step this alternative's attractiveness benefits from including non-diagnostic information between its pros and cons. Sales persons can rely on this principle to increase the success of sales conversations. Companies offering products in online shops could use this principle to increase sales by using appropriate techniques that follow the suggested mode of sequential information presentation. 


\section{Problemstellung}

\subsection{Alternativenweise und attributweise Informationspräsentation}

Ein Verkäufer kann den Ablauf eines Verkaufsgesprächs steuern. Er kann dem Kunden nacheinander verschiedene Produkte vorstellen, deren Eigenschaften beschreiben und den jeweiligen Preis benennen. In diesem Fall präsentiert er die Informationen alternativenweise. Er könnte die Produkte allerdings auch je Merkmal erklären - z. B. zuerst die technischen Eigenschaften aller Produkte erläutern, dann auf die Garantien für alle Produkte eingehen, sodann auf Finanzierungsmöglichkeiten hinweisen und im letzten Schritt die Preise für alle Produkte angeben. In diesem Fall wählt er eine attributweise Informationspräsentation. Wenn der Kunde einen Online-Shop aufsucht, könnten Programme ebenfalls steuern, in welcher Reihenfolge Kunden Informationen verarbeiten, wie sie diese kombinieren und somit letztendlich wofür sie sich entscheiden, so dass Anbieter durch eine geschickte Lenkung der Sequenz, in der die Informationseinheiten bereit gestellt werden, Gewinne beeinflussen könnten. Levav et al. (2010) zeigten dies am Beispiel eines Car-Configurators eines Pkw-Herstellers in Feldexperimenten.

\subsection{Möglichkeiten der attributweisen Informationspräsentation im Internet}

Auf der Website von Zeit Online (2012) kann der potenzielle Student zum Thema Hochschulauswahl vorab auswählen, welche Fachrichtung er studieren möchte und welchen Abschlusstyp er anstrebt. Nach dieser Vorauswahl kann er ein erstes Merkmal (z. B. „Ausstattung ") anklicken, und das Programm liefert die Information über diesbezüglich gute und schlechtere Standorte in einer Art Kreisdiagramm. Dann kann er das nächste Merkmal (z. B. „Internationale Ausrichtung“) eingeben, und das Programm gibt für dieses Merkmal die entsprechende Information aus usw. Auf diese Art und Weise verarbeitet der Rezipient Information über alle ausgewählten Standorte attributweise.

Wahlbürger können mit Hilfe von als „Wahl-O-Mat“ bezeichneten Online-Programmen zu einem bestimmten, persönlich sehr wichtigen Thema eine eigene politische Position eingeben und dann nach Überspringen der vielfältigen weiteren Themen feststellen, welche Parteien diese Position teilen. Dann können sie ihre Position zum nächst-wichtigen, individuell ausgewählten Thema angeben und wieder nach Überspringen der weiteren Themen die Information abrufen, welche Parteien die Position unterstützen. Auf diese Weise kann der Wahlbürger leicht attributweise Information über verschiedene Parteien abrufen. Diese Vorgehensweise wird der Wahlbürger insbesondere dann wählen, wenn er sich nur für vergleichsweise wenige Themen, hinsichtlich derer sich die Parteien unterscheiden könnten, interessiert.

Avatare in Online-Shops wie z. B. „Anna“ auf der Website des Online-Shops von Ikea (2012), mit denen Kunden interagieren, könnten die Reihenfolge der Attributsequenz ebenfalls beeinflussen: Sinngemäss könnte eine Interaktion mit einem virtuellen Gesprächspartner wie folgt anlaufen: „Du willst etwas über ... wissen? Darf ich dir zunächst aufzeigen, welche (Merkmal 1) die ... haben?“ “... „Darf ich dir jetzt zeigen, wie sich die ... hinsichtlich (Merkmals 2) unterscheiden?" etc.

Die Reihenfolge der Merkmale in diesen Sequenzen könnte die Entscheidung der potenziellen Studenten, der Wahlbürger und der Konsumenten beeinflussen. Sind Produkte auszuwählen und unterscheiden sich diese hinsichtlich der Deckungsbeiträge, beeinflusst die gewählte Reihenfolge der Attribute möglicherweise den Gewinn für das Unternehmen. 
Beispiele aus der Praxis, wonach über Online-Portale der oben beschriebenen Art die Reihenfolge der Attribute bereits gezielt gesteuert wird, sind uns nicht bekannt. Allerdings wäre diese Technik leicht einsetzbar, und daher ist Wissen über erzielbare Reihenfolge-Effekte von Interesse für die Praxis.

\subsection{Möglichkeiten der attributweisen Informationspräsentation im Verkaufsgespräch}

Carlson et al. (2006) und Carlson et al. (2009) verweisen darauf, dass Verkäufer im persönlichen Gespräch Informationen über die Wahlmöglichkeiten häufig attributweise zur Verfügung stellen. Angenommen, ein Kunde lässt sich in einem Reisebüro über Möglichkeiten, in einem exotischen Land Urlaub zu machen, beraten, und der Verkäufer kann z. B. drei Pauschalreisen anbieten, von denen der Kunde eine buchen könnte. Der Verkäufer könnte für alle drei Angebote zunächst die Orte der Hotels vorstellen, dann für alle drei Angebote die Umgebung, die der Kunde jeweils besichtigen kann, sodann für alle drei Angebote die Ausstattung der Hotels und des Weiteren für alle drei Angebote die Freizeitmöglichkeiten. Diese attributweise Vorgehensweise erleichtert es dem Kunden, bereits während der Informationspräsentation Vergleiche anzustellen. Würde der Verkäufer anstelle der attributweisen die alternativenweise Informationspräsentation wählen, hätte der Kunde erhebliche Schwierigkeiten, die Informationen zur zuerst präsentierten Alternative zu bewerten, während diese vorgestellt werden, und sich im weiteren Fortgang während der Präsentation der weiteren Alternativen zu erinnern, welche Attributausprägungen die zuvor präsentierten Alternativen aufweisen. Der Kunde könnte den Verkäufer sogar auffordern, die Informationen attributweise vorzustellen, damit er leichter Vergleiche anstellen kann. Auch im persönlichen Verkaufsgespräch besteht die Möglichkeit, dass der Verkäufer die Reihenfolge der Attribute gezielt steuert.

In Verkaufsleitfäden wird empfohlen, im Fall, dass ein Anbieter am Verkauf des vergleichsweise teuren Produkts besonders interessiert ist, den hohen Preis als Produktnachteil erst am Ende des Verkaufsgesprächs zu thematisieren und zeitlich zuvor auf die Produktvorteile gegenüber den billigeren Wahlmöglichkeiten einzugehen (z. B. Bänsch 2006, 78). Gleichwohl diese Regel intuitiv einleuchtet, ist unseres Wissens noch nicht untersucht worden, ob sie tatsächlich den gewünschten Effekt auslöst oder ob zusätzliche Bedingungen zu erfüllen sind, damit der Effekt auftritt.

\subsection{Zielsetzung der Studie und Vorgehensweise}

Die hier behandelte Thematik ist dem Gebiet der Erforschung von Reihenfolge-Effekten zuzuordnen. Zugegebenermassen ist dieses Gebiet sehr breit, und manche Teilgebiete, über die später ein kurzer Überblick gegeben wird, sind bereits intensiv erforscht.

Die Wirkung der attributweisen Informationspräsentation im Fall von Wahlmöglichkeiten ist verglichen mit anderen Teilgebieten zum Thema Reihenfolge-Effekt hingegen bislang wenig erforscht worden (Carlson et al. 2006; Malaviya/Sternthal 2009). Wenn man von Sonderaspekten, die in den beiden eben genannten Studien behandelt werden, absieht, fand die bisherige Forschung keinen Effekt der Reihenfolge, in der Attribute präsentiert werden, auf den Ausgang der Entscheidung.

Das Ziel dieser Abhandlung ist es zu zeigen, wie man im Fall der attributweisen Informationspräsentation einen Reihenfolge-Effekt herbeiführen kann, wenn Personen am Ende der Informationspräsentation eine Entscheidung treffen. Unsere These lautet, dass ein Rei- 
henfolge-Effekt entsteht, wenn zwischen der Darstellung von Vor- und Nachteilen nichtdiagnostische Information eingebunden wird. Information ist nicht-diagnostisch, wenn sie sich nicht eignet, die Wahlmöglichkeiten zu unterscheiden. Wir betrachten speziell Merkmale, hinsichtlich derer die Wahlmöglichkeiten identische Ausprägungen annehmen, als nicht-diagnostische Information. Malaviya/Sternthal (2009) bezeichnen diese Information als „parity features“. Nicht-diagnostische Information ist nicht zwangsläufig irrelevant. Alle Pkw haben vier Räder; insofern ist die Information, ein bestimmter Pkw habe vier Räder, nicht-diagnostisch. Dass ein Auto vier Räder hat, ist jedoch nicht irrelevant für das Funktionieren eines Pkws.

Wir entwickeln die Forschungsrichtung also weiter, indem wir prüfen, ob sich durch die Einbindung von nicht-diagnostischer Information im Fall der attributweisen Informationspräsentation ein systematischer Reihenfolge-Effekt ergibt. Diese Möglichkeit ist unserem Wissen nach noch nicht überprüft worden. Diese Abhandlung zielt also nicht darauf ab, Schwächen früherer Studien aufzudecken und zu beseitigen, sondern eine neue Möglichkeit zu testen, einen Reihenfolge-Effekt herbeizuführen.

Diese Abhandlung ist im weiteren Fortgang wie folgt gegliedert. Nach der Definition des untersuchten Effekts und seinen Ausprägungen (Primacy-Effekt, Recency-Effekt) wird ein knapper Überblick über die Hauptströmungen der Forschung auf den Gebiet des Reihenfolge-Effekts geboten, und wichtige Erkenntnisse dieser Richtungen werden aufgezeigt. Anschliessend werden zwei Theorien vorgestellt (Biased-Hypothesis-Testing, Adaptationsniveautheorie), mittels derer sich jeweils Prognosen ableiten lassen, dass nicht-diagnostische Information einen Reihenfolge-Effekt bewirkt. Allerdings sind diese Hypothesen widersprüchlich. Biased-Hypothesis-Testing sagt einen Primacy-Effekt, die Adaptationsniveautheorie sagt einen Recency-Effekt voraus. Daher wird nach einer Bedingung gesucht, unter der der eine oder andere Effekt überwiegen könnte. In drei Experimenten werden die resultierenden Hypothesen überprüft. Abschliessend werden die Folgerungen aus diesen Erkenntnissen diskutiert.

\section{Definition des Reihenfolge-Effekts und Hauptströmungen der Forschung}

\subsection{Definition und Abgrenzungen}

Hogarth/Einhorn $(1992,3)$ definieren den Reihenfolge-Effekt wie folgt: „There are two pieces of evidence, A and B. Some subjects express an opinion after seeing the information in the order A-B; others receive the information in the order B-A. An order effect occurs when opinions after A-B differ from those after B-A.“ Die Forschung beschränkt sich jedoch nicht nur auf „opinions“, sondern betrachtet auch andere Responsevariablen. Allgemeiner formuliert tritt ein Reihenfolge-Effekt nicht nur auf, wenn die Variation der Reihenfolge von Informationseinheiten einen Einfluss darauf hat, wie bewertet und entschieden wird, sondern auch darauf einen Einfluss hat, woran sich Personen erinnern (Pieters/ Bijmolt 1997; Terry 2005; Raanaas/Magnussen 2006). In diesem Zusammenhang werden zwei Ausprägungen des Reihenfolge-Effekts unterschieden, die als Primacy-Effekt und Recency-Effekt bezeichnet werden (Anderson 1981, 145; Highhouse/Gallo 1997, 31; Petty et al. 2001, 332; Igou/Bless 2003, 91). Nach Kardes/Herr (1990, 541) liegt der Primacy-Effekt vor, wenn „information appearing early in a sequence has a stronger effect on judgment than does subsequent information", wohingegen der Recency-Effekt vorliegt, wenn „later items dominate earlier items“. Anders formuliert: Wenn Informationseinheiten al- 
lein deshalb, weil sie am Anfang bzw. am Ende einer sequentiellen Informationspräsentation enthalten sind, ein höheres kognitives Gewicht erlangen, besteht der Primacy- bzw. der Recency-Effekt.

Der nachfolgende Überblick geht auf Hauptströmungen in der Forschung zu Reihenfolge-Effekten ein. Wir teilen danach ein, zu wie vielen Meinungsobjekten Information geboten wird und welche Art von Informationseinheiten in ihrer Reihenfolge variiert wird. In Abbildung 1 wird die hier untersuchte Richtung (attributweise Informationspräsentation) von anderen, hier nur in Form eines kurzen Literaturüberblicks behandelten Richtungen abgegrenzt.

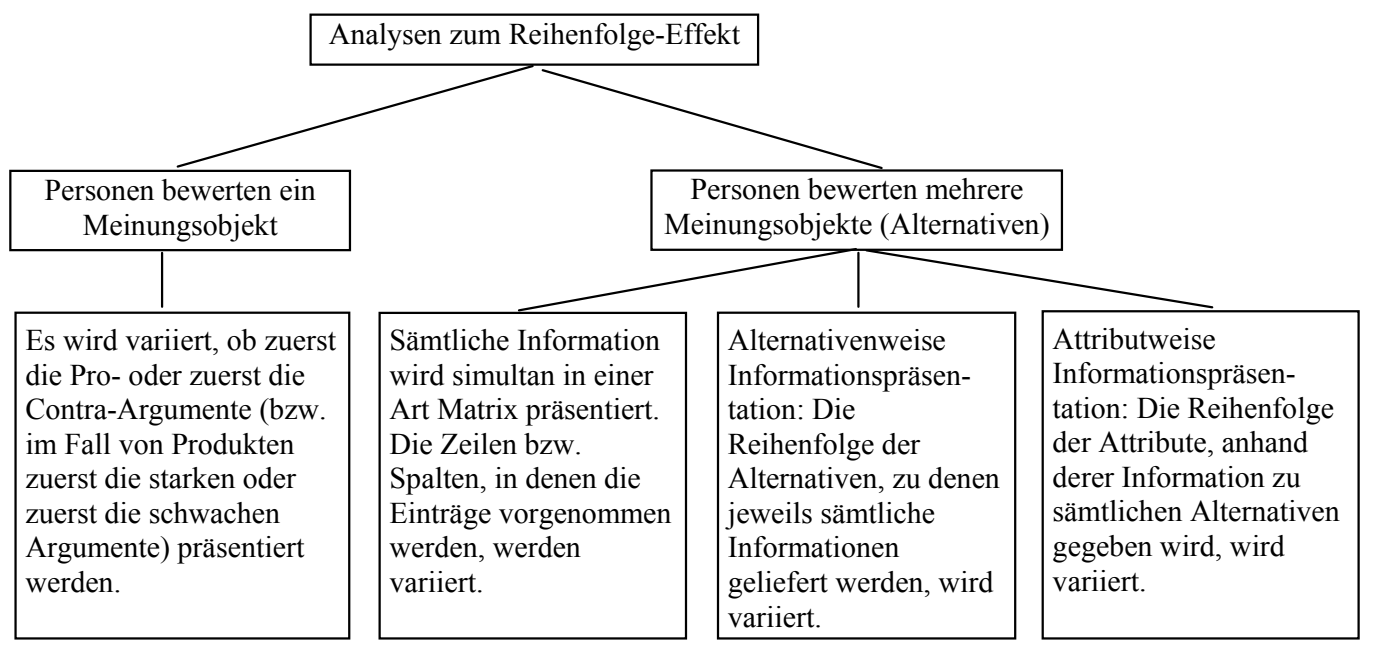

Abbildung 1: Überblick über Forschungsrichtungen zum Reihenfolge-Effekt

\subsection{Variation der Reihenfolge von Information im Fall eines einzelnen Meinungsobjekts}

In der Literatur findet sich vielfältige Forschung zu folgender Situation: Personen sollen ein Urteil über ein Meinungsobjekt (z. B. über ein politisches Thema oder über eine fremde Person) abgeben, und es wird variiert, ob zuerst die vorteilhafte oder erst die nachteilige Information gegeben wird. Die diesbezüglich klassische Studie stammt von Lund (1925, 184 ff.). Er präsentierte Probanden einen Text zum Meinungsobjekt „Should all men have equal political rights?" und variierte, ob im Text zuerst die Pro- oder zuerst die ContraArgumente aufgeführt waren (zu ähnlichen Fragestellungen vgl. z. B. Asch 1946; Hovland/ Mandel 1957; Lana 1961). Hogarth/Einhorn (1992) boten einen Literaturüberblick über 76 Studien dieser Art und ermittelten, dass Bewertungen - für den Fall, dass Personen erst am Ende der Informationspräsentation bewerten - tendenziell positiver ausfallen, wenn zuerst auf die Pro-Argumente hingewiesen wird (Primacy-Effekt). Haugtvedt/Wegener (1994) berichten ein weiteres wichtiges Ergebnis. Für Personen, denen das Meinungsobjekt wichtig ist, stellten sie stärkere Zustimmungen im Fall der Erst-Pro-Dann-Contra-Argumente-Reihenfolge fest (Primacy-Effekt). Für Personen, denen das Meinungsobjekt unwichtig ist, ermittelten sie hingegen stärkere Zustimmungen im Fall der Erst-Contra-DannPro-Argumente-Reihenfolge (Recency-Effekt). 
Derartige Studien existieren auch für Produkte als Meinungsobjekte. Hier erlangt eine Personengruppe zuerst Kenntnis über die die starken Argumente und eine andere Personengruppe zuerst Kenntnis über die schwachen Argumente für das zu bewertende Produkt (z. B. Gürhan-Canli 2003; Igou/Bless 2003; Tavassoli/Lee 2004; Bond et al. 2007; Biswas et al. 2009). Argumente sind stark, wenn sie Gedanken des Konsumenten auslösen, wonach das Produkt gegenüber Konkurrenzprodukten überlegen ist. Sie sind schwach, wenn sie Gedanken auslösen, wonach das Produkt hinsichtlich des thematisierten Nutzens nicht überlegen ist (Petty et al. 1983, 139; Petty/Cacioppo 1986, 32). Biswas et al. $(2009,96)$ verwendeten in ihrer Studie z. B. den Hinweis „hoch angesehene Marke“ als ein starkes Argument und den Hinweis „gute Garantie“ als ein schwaches Merkmal für eine zu bewertende Armbanduhr. Nicht jede Armbanduhr stammt von einer renommierten Marke, aber jede Armbanduhr besitzt eine „gute Garantie“. Letzteres Merkmal ist jedoch kein Contra-Argument, das gegen den Kauf dieses Produkts spricht, es spricht lediglich nicht dafür. Igou/Bless (2003) stellten fest, dass Produkte positiver bewertet werden, wenn erst die starken und dann die schwachen Argumente dargeboten werden (Primacy-Effekt). Sie begründen diese Beobachtung damit, dass Konsumenten meinen, die Anbieter würden ihre starken Argumente gleich zu Beginn einer Informationspräsentation anführen. Weitere Autoren untersuchten, ob dennoch Moderatorvariablen existieren, von denen die Vorteilhaftigkeit der verschiedenen Reihenfolgen abhängt. Biswas et al. (2009) zeigten, dass es in dem Fall, in dem die Aufmerksamkeit der Personen während der Informationsaufnahme gezielt vermindert wird, von Vorteil ist, die Argumente in der Reihenfolge „erst die schwachen, dann die starken Argumente" zu liefern (Recency-Effekt). Ist ihre Aufmerksamkeit hingegen nicht vermindert, wird ein Produkt positiver bewertet, wenn die Argumente in der Reihenfolge „erst die starken, dann die schwachen Argumente“ dargeboten werden (Primacy-Effekt).

\subsection{Variation von Zeilen und Spalten in einer Informationsmatrix}

Manche Autoren zeigen Personen eine Übersicht, z. B. eine Tabelle mit Zahleneinträgen und/oder Bildern, in der sämtliche Information enthalten ist. Für den Fall, dass Information zu zwei Optionen und vier Merkmalen existiert, würde eine Übersicht wie in Tabelle 1 skizziert aussehen.

\begin{tabular}{lll}
\hline & Alternative A & Alternative B \\
\hline Merkmal 1 & $\mathrm{x}_{1 \mathrm{~A}}$ & $\mathrm{x}_{1 \mathrm{~B}}$ \\
Merkmal 2 & $\mathrm{x}_{2 \mathrm{~A}}$ & $\mathrm{x}_{2 \mathrm{~B}}$ \\
Merkmal 3 & $\mathrm{x}_{3 \mathrm{~A}}$ & $\mathrm{x}_{3 \mathrm{~B}}$ \\
Merkmal 4 & $\mathrm{x}_{4 \mathrm{~A}}$ & $\mathrm{x}_{4 \mathrm{~B}}$ \\
\hline
\end{tabular}

Tabelle 1: Vollständige Information in einer Tabelle

In diesem Fall können zum einen die Zeilen, in denen Informationen über Merkmale enthalten sind, variiert werden. Abweichend von den Einträgen in Tabelle 1 kann also, von oben beginnend, der Eintrag von Merkmal 4 in der ersten Zeile, dann der Eintrag von Merkmal 3 in der zweiten Zeile etc. erfolgen. Kjoer et al. (2006) stellten fest, dass die Preissensitivität von Personen vergleichsweise hoch ist, wenn der Preis in der untersten Zeile enthalten ist (Recency-Effekt). Zum anderen können die Spalten variiert werden. Leesch et 
al. (2010) ermittelten, dass ein Produkt einen Präferenzvorteil aus dem Tatbestand erlangt, dass es in der linken Spalte (dies wäre in Tabelle 1 die Alternative A) beschrieben wird. Diese Autoren ermittelten auch einen Präferenzvorteil für eine Option, wenn diese in einer Liste ganz oben angesiedelt ist.

\subsection{Variation der Reihenfolge der präsentierten Alternativen im Fall mehrerer Optionen}

Des Weiteren beschäftigen sich Autoren mit der Frage, wie Personen bewerten und entscheiden, wenn Informationen zu verschiedenen Wahlmöglichkeiten alternativenweise präsentiert werden, z. B. zuerst alle Informationseinheiten für A und dann alle für B oder umgekehrt erst alle Informationen zu B und dann alle zu A (z. B. Scarpi 2004). In diesem Zusammenhang ist es interessant, dass einige Autoren einen Reihenfolge-Effekt feststellten, der von der generellen Attraktivität der Optionen abhing. Biswas et al. (2010) beispielsweise liessen Probanden zwei hochwertige Biere in einem Produkttest probieren, und 65\% bevorzugten das als zweites getestete Bier (Recency-Effekt). Liessen sie hingegen zwei weniger hochwertige Biere verkosten, bevorzugten $69 \%$ das als erstes verkostete Bier (Primacy-Effekt). Denselben Effekt ermittelten Bruine de Bruin/Keren (2003) am Beispiel der Auswahl von Blind-Date-Partnern und Li/Epley (2009) für weitere Kategorien.

\subsection{Variation der Reihenfolge der präsentierten Merkmale im Fall mehrerer Optionen}

Liegen mehrere Wahlmöglichkeiten und Merkmale vor, kann die Information auch attributweise präsentiert werden, z. B. zuerst Information zu Merkmal 1 für alle Optionen, dann Information zu Merkmal 2 für alle Optionen etc. Der Effekt dieser Manipulation ist bislang kaum erforscht worden.

Einige Autoren präsentierten zwar Information attributweise, sie variierten jedoch deren Reihenfolge nicht (z. B. Kardes/Herr 1990; Russo et al. 1996; Russo et al. 1998; Russo et al. 2000; Carlson/Pearo 2004; Russo et al. 2008; Polman 2010; Russo/Chaxel 2010). Carlson et al. (2009) untersuchten auf diese Art und Weise, wie viele Personen nach jedem einzelnen Schritt, d. h. nach Merkmal 1, nach Merkmal 2 etc. die jeweiligen Alternativen bevorzugen. Malaviya/Sternthal (2009) variierten die Reihenfolge der attributweise präsentierten Information und untersuchten, ob eine Option A, die einer Option B gegenüber hinsichtlich aller diagnostischen Informationen überlegen ist, noch positiver bewertet wird, wenn nicht-diagnostische Information hinzukommt und diese bereits zu Beginn oder erst am Ende der Informationspräsentation aufgeführt wird. Dieser Spezialaspekt wird hier nicht vertieft.

Eine Studie von Carlson et al. (2006) hat die höchste Ähnlichkeit mit den hier vorgenommenen Experimenten. Die Autoren präsentierten Testpersonen zwei Wintermäntel, A und B, als Wahlmöglichkeiten und beschrieben sie anhand von sechs Merkmalen, auf deren Inhalt sie im Beitrag nicht eingehen. Darunter befanden sich fünf Merkmale, die Vorteile für A beinhalteten; das sechste Merkmal beschrieb einen Vorteil für B. Die Testpersonen wurden in zwei Gruppen eingeteilt (ca. 57 pro Gruppe). Die eine Gruppe sah den Nachteil von A an der vierten Position der attributweisen Informationspräsentation (Reihenfolge 1), die andere Gruppe sah den Nachteil von A gleich zu Beginn der Informationspräsentation (Reihenfolge 2). Das Ergebnis dieser Studie war, dass sich im Fall der Reihenfolge $180 \%$ der Testteilnehmer und im Fall der Reihenfolge 2 65\% der Testteilnehmer, die am Ende der Informationspräsentation eine Wahl treffen mussten, für Option A ent- 
schieden. Deskriptiv betrachtet entstand ein Primacy-Effekt, die Differenz dieser Anteile erwies sich jedoch nicht als signifikant. Sondereffekte traten auf, wenn die Personen nach jeder einzelnen Informationseinheit, d. h. insgesamt sechsmal, Bewertungen abgeben mussten.

Wir greifen diese Art von Studie auf und untersuchen, ob sich doch ein Reihenfolge-Effekt ergibt, wenn zwischen der Darstellung der Vor- und Nachteile nicht-diagnostische Information eingebunden wird. Carlson et al. (2006) hatten, wie eben erläutert, auf die Berücksichtigung von nicht-diagnostischer Information verzichtet.

\section{Entscheidungssituation und theoretische Überlegungen}

\subsection{Entscheidungssituation}

Um die in den nachfolgenden Abschnitten vorgestellten theoretischen Überlegungen illustrieren zu können, konstruieren wir vorab in Form eines Gedankenspiels eine einfache Entscheidungssituation. Man stelle sich vor, dass zwei Wahlmöglichkeiten, A und B, existieren, zwischen denen eine Wahl zu treffen ist. Des Weiteren stelle man sich vor, dass zu jeder Alternative Informationen über zwei oder mehrere Merkmale vorliegen. Für ein erstes Szenario sei angenommen, dass A bei Merkmal 1 einen Vorteil gegenüber B (Notation: $\left.x_{1 \mathrm{~A}}>x_{1 \mathrm{~B}}\right)$ und $\mathrm{B}$ bei Merkmal 2 einen Vorteil gegenüber A hat $\left(x_{2 \mathrm{~A}}<x_{2 \mathrm{~B}}\right)$. Information kann entweder so präsentiert werden, dass zuerst auf den Vorteil von A aufmerksam gemacht und anschliessend auf den Vorteil von B hingewiesen wird. Umgekehrt könnte man auch erst auf den Vorteil von B verweisen, bevor der Vorteil von A aufgezeigt wird. Für ein zweites Szenario stelle man sich vor, dass die Information hinzukommt, dass sich A und $\mathrm{B}$ hinsichtlich eines dritten und vierten Merkmals nicht unterscheiden $\left(x_{3 \mathrm{~A}}=x_{3 \mathrm{~B}}\right.$ und $\left.x_{4 \mathrm{~A}}=x_{4 \mathrm{~B}}\right)$. Dabei handelt es sich um zwei hinsichtlich der Entscheidung zwischen A und $\mathrm{B}$ nicht-diagnostische Informationseinheiten. Diese Information kann zwischen der Präsentation von Merkmal 1 und Merkmal 2 eingebunden werden. In Kombination mit der Festlegung, ob zuerst der Vorteil von A oder zuerst der Vorteil von B vorgestellt wird, resultieren die in Abbildung 2 dargestellten vier Fälle, wie Informationseinheiten attributweise zur Verfügung gestellt werden können.

\section{erste \\ zweite \\ dritte \\ vierte \\ Information \\ Information \\ Information \\ Information}

Fall 1:

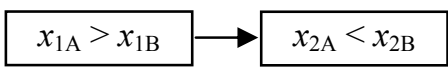

Fall 2:

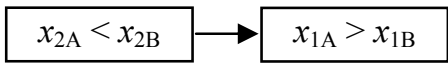

Fall 3:

$x_{1 \mathrm{~A}}>x_{1 \mathrm{~B}} \rightarrow x_{3 \mathrm{~A}}=x_{3 \mathrm{~B}} \rightarrow x_{4 \mathrm{~A}}=x_{4 \mathrm{~B}} \rightarrow x_{2 \mathrm{~A}}<x_{2 \mathrm{~B}}$

Fall 4:

$x_{2 \mathrm{~A}}<x_{2 \mathrm{~B}} \rightarrow x_{3 \mathrm{~A}}=x_{3 \mathrm{~B}} \rightarrow x_{4 \mathrm{~A}}=x_{4 \mathrm{~B}} \rightarrow x_{1 \mathrm{~A}}>x_{1 \mathrm{~B}}$

Abbildung 2: Verschiedene Reihenfolgen der attributweisen Informationspräsentation 


\subsection{Biased-Hypothesis-Testing und Adaptationsniveautheorie}

Zunächst wird theoretisch hergeleitet, warum die nicht-diagnostische Information einen Primacy-Effekt auslöst. Als Grundlage für diese Vermutung kommt die Theorie des Biased-Hypothesis-Testing in Betracht. Diese Theorie wurde von Snyder/Swann (1978) und Snyder/Cantor (1979) entwickelt. Sie besagt, dass Personen eine Ausgangshypothese zu einem Sachverhalt bilden und nachfolgend gebotene Information dahingehend überprüfen, ob sie zu dieser Hypothese passt, anstatt zu überprüfen, ob sie ihr widerspricht. Im Grundlagenexperiment dieser Autoren lasen Probanden einen Text über eine Frau, die zur Hälfte durch introvertierte Eigenschaften und zur Hälfte durch extrovertierte Eigenschaften beschrieben war. Der eine Teil der Probanden sollte später urteilen, ob sich diese Frau für den extrovertierten Beruf eines Immobilienmaklers eignet (Hypothese); die Auskunftspersonen führten vergleichsweise mehr Gründe, die dafür sprachen, an. Der andere Teil der Probanden sollte bewerten, ob sie sich für den introvertierten Beruf einer Bibliotheksangestellten eignet (Hypothese), und auch diese Personen führten mehr Pro-Argumente auf. Biased-Hypothesis-Testing heisst, dass Personen dazu neigen, Information so zu interpretieren, dass sie zur Ausgangshypothese passt. Personen tendieren also dazu, eine für sie neue Information so aufzufassen, dass diese die bisherige Meinung oder eine vorgegebene Hypothese bestätigt. Diese Theorie wurde in diversen anderen Bereichen erfolgreich getestet. Beispielsweise zeigten $\mathrm{Hoch} / \mathrm{Ha}$ (1986), dass Konsumenten dazu neigen, die in einer Werbung enthaltene Information so zu interpretieren, dass sie die Annahme stützt, dass das beworbene Produkt ein gutes Produkt ist, da Konsumenten die Ausgangshypothese haben, dass das Produkt gut sei - ansonsten würde es nicht beworben. Auch für den Fall von Information, von der nicht klar ist, ob sie für oder gegen den Kauf eines bestimmten Produkts spricht (ambigue Information), wurde festgestellt, dass Konsumenten dazu tendieren, diese Information im Sinne der Bestätigung der Ausgangshypothese, es handle sich um ein gutes Produkt, zu interpretieren (Ha/Hoch 1989, 359). Darley/Gross (1983) belegten diese Theorie in einer Studie, in der Probanden die Leistungsfähigkeit einer Schülerin auf der Basis einer Ausgangshypothese bewerten mussten. Sanbonmatsu et al. (1997) erklärten den Tatbestand, dass Personen die Wahrscheinlichkeit, dass ein bestimmter Bewerber um eine Arbeitsstelle diese Arbeitsstelle tatsächlich erhält, überschätzen, mit BiasedHypothesis-Testing. Posavac et al. (2010) fanden Belege für diese Theorie in einer Studie, in der Probanden bewerten sollten, welches Produktdesign für ein neues Produkt vorteilhaft sei. Meyvis/Janiszewski (2002, 620) begründen dieses Phänomen damit, dass die Bestätigung einer Hypothese mit geringerem kognitiven Aufwand verbunden ist als ihre Ablehnung.

Wir übertragen diese Theorie auf den hier betrachteten Fall. Aus ihr ist zu folgern, dass sukzessiv verfügbar gemachte Information so verarbeitet wird, dass Personen Schritt für Schritt testen, ob eine jeweils vorher gebildete Hypothese gültig ist, anstatt zu prüfen, ob diese nicht stimmt. Dies führt dazu, dass Personen möglichst wenige inkonsistente Urteile hinsichtlich eines Sachverhalts bilden müssen (Carlson et al. 2006; Russo et al. 2008).

Angenommen, die erste Informationseinheit drückt einen Vorteil der Alternative A gegenüber der Alternative B aus $\left(x_{1 \mathrm{~A}}>x_{1 \mathrm{~B}}\right)$. Hieraus entsteht „A ist vorteilhaft“ als erster Eindruck, der als die Ausgangshypothese für die weitere Informationsverarbeitung dient. Weiterhin angenommen, die zweite Informationseinheit sei nicht-diagnostisch $\left(x_{2 \mathrm{~A}}=x_{2 \mathrm{~B}}\right)$. Diese Information bringt zum Ausdruck, dass die Alternativen hinsichtlich des zweiten Merkmals objektiv gleichwertig sind. Um das anfänglich gebildete Urteil aufrechtzuerhal- 
ten, d. h. die Ausgangshypothese zu stützen, kann die zweite Information wie folgt interpretiert werden: „A ist nicht schlechter als B“. Also gibt es auch nach dem Kontakt mit zwei Merkmalen keinen Grund anzunehmen, warum Alternative A nicht die vorteilhafte sei. Des Weiteren unterstellt, die dritte Informationseinheit sei ebenfalls nicht-diagnostisch $\left(x_{3 \mathrm{~A}}=x_{3 \mathrm{~B}}\right)$. Wird diese Information dahingehend bewertet, ob sie die bislang gebildete Meinung „A ist besser als B“ stützen kann, so wird dies die Person bejahen. Sogar nach dem Kontakt mit drei Merkmalen gibt es im hier konstruierten Beispiel keinen Grund anzunehmen, warum Alternative A nicht die vorteilhafte sein sollte. Schliesslich unterstellt, das vierte Merkmal enthalte einen Nachteil von A gegenüber B $\left(x_{4 \mathrm{~A}}<x_{4 \mathrm{~B}}\right)$. Nach drei Informationen, die den Eindruck erweckt und verfestigt hatten, A sei die bessere Option, folgt nun erstmals eine Information, die mit dem bisherigen Urteil inkonsistent ist. Nun stelle man sich vor, dass die Vor- und Nachteile in der umgekehrten Reihenfolge präsentiert werden $\left(x_{4 \mathrm{~A}}<x_{4 \mathrm{~B}} \rightarrow x_{3 \mathrm{~A}}=x_{3 \mathrm{~B}} \rightarrow x_{2 \mathrm{~A}}=x_{2 \mathrm{~B}} \rightarrow x_{1 \mathrm{~A}}>x_{1 \mathrm{~B}}\right)$. Nach drei Informationen, die die Überzeugung herbeigeführt hatten, B sei die vorteilhafte Alternative, entsteht erstmals ein Kontakt mit einer einzelnen, inkonsistenten Information. Wenn sich eine Person schlicht an der Anzahl der Informationen orientiert, die mit der aufgrund der ersten Informationseinheit gebildeten Ausgangshypothese nicht widersprüchlich sind, oder eine einzelne inkonsistente Information vernachlässigt oder gering gewichtet, entsteht im Beispiel durch die Einbindung von nicht-diagnostischer Information ein Präferenzvorteil für die Option A. Nach dieser Theorie ist also das Auftreten des Primacy-Effekts zu erwarten.

Es kann aber auch argumentiert werden, dass die nicht-diagnostische Information einen Recency-Effekt auslöst. Der Grund ist, dass die nicht-diagnostische Information zu einer Assimilation der Alternativen führt. Merkmale, hinsichtlich derer sich die Optionen nicht unterscheiden, können bewirken, dass sich die wahrgenommene Ähnlichkeit der Optionen erhöht. Wird je Schritt das Averaging-Prinzip angewendet, entsteht ein Präferenzvorteil für die Alternative B, deren Vorteil am Ende der Informationspräsentation ersichtlich wird. Als theoretische Begründung kann die Adaptationsniveautheorie von Helson (1964) herangezogen werden, wonach sich ein neues Urteil aus dem bisherigen Urteil und der jeweils neuen Information ergibt: $R_{\mathrm{t}}=\lambda R_{\mathrm{t}-1}+(1-\lambda) x_{\mathrm{t}}(R$ : Urteil, $x$ : neue Information, $t$ : Zeitpunkt, $0<\lambda<1)$. Diese Theorie wurde ursprünglich entwickelt, um psychophysikalische Effekte vorherzusagen (Helson 1947; Kröner 1974; Sitmalidis 1976; Sarris/Lauterbach 1980). Sie wird aber auch herangezogen, um den so genannten Dilution-Effekt zu erklären, der besagt, dass nicht-diagnostische Information nicht ignoriert wird, sondern mit einem positiven Gewicht in die Urteile einfliesst, weswegen extreme Urteile weniger extrem ausfallen (z. B. Troutman/Shanteau 1977; Nisbett et al. 1981). Bereits Hogart/Einhorn (1992, 10) vermuteten, dass der beschriebene Prozess einen Reihenfolge-Effekt auslösen kann.

Diese Theorie kann ebenfalls auf die Problemstellung angewendet werden. Zur Erklärung des postulierten Effekts wird ein Zahlenbeispiel für $\lambda=1 / 2$ gebildet, und die Zwischen- und Endergebnisse werden in Abbildung 3 skizziert. Man stelle sich zunächst vor, dass die erste Informationseinheit aus $x_{1 \mathrm{~A}}=7$ und $x_{1 \mathrm{~B}}=1$ besteht; diese ergeben das anfängliche Urteil $\left(R_{1 \mathrm{~A}}=7, R_{1 \mathrm{~B}}=1\right)$. Die nächste Information sei $x_{2 \mathrm{~A}}=x_{2 \mathrm{~B}}=4$. Averaging führt zum Urteil $R_{2 \mathrm{~A}}=5,5$ und $R_{2 \mathrm{~B}}=2,5$ für $\mathrm{B}$. Die folgende Information sei ebenfalls $x_{3 \mathrm{~A}}$ $=x_{3 \mathrm{~B}}=4$. Averaging zwischen 5,5 und 4 führt zu $R_{3 \mathrm{~A}}=4,75$ und Averaging zwischen 2,5 und 4 führt zu $R_{3 \mathrm{~B}}=3,25$. Die letzte Information sei $x_{4 \mathrm{~A}}=1$ und $x_{1 \mathrm{~B}}=7$. Averaging zwischen 4,75 und 1 bewirkt $R_{4 \mathrm{~A}}=2,875$ und Averaging zwischen 3,25 und 7 führt zu $R_{4 \mathrm{~B}}=$ 
5,125. Eine andere Gewichtung mit $\lambda$ ändert das Ergebnis nicht systematisch. Die Option A erleidet nach dieser Theorie einen Präferenznachteil, wenn zu Beginn ihr Vorteil und am Ende ihr Nachteil aufgezeigt wurde. Dreht man die Reihenfolge um (erst der Vorteil von B, am Ende der Nachteil von B), erleidet Option B einen Präferenznachteil. Verarbeiten Personen sukzessive Information gemäss dem in der Adaptationsniveautheorie postulierten Averaging-Prinzip, entsteht der Recency-Effekt.

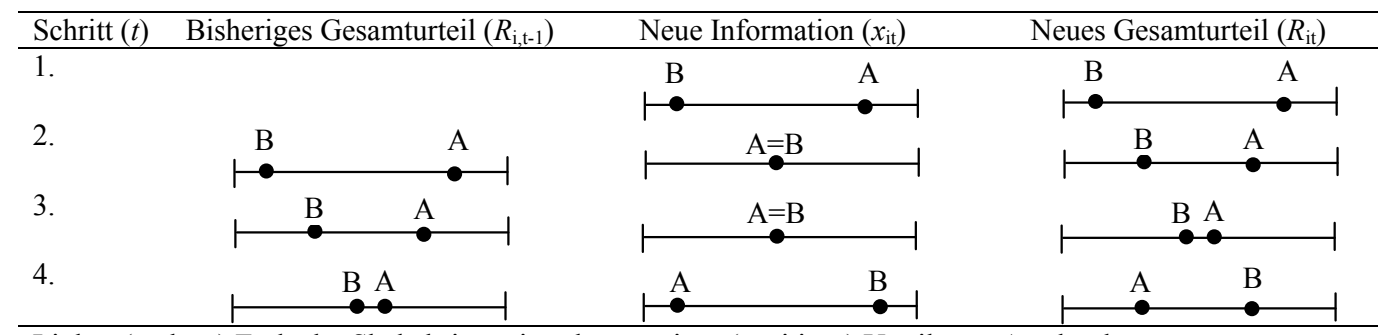

Linkes (rechtes) Ende der Skala bringt ein sehr negatives (positives) Urteil zum Ausdruck.

Abbildung 3: Veranschaulichung des durch Averaging entstehenden Recency-Effekts

Diese beiden weithin etablierten Theorien, Biased-Hypothesis-Testing and die Adaptationsniveautheorie, liefern konträre Vorhersagen, ob nicht-diagnostische Information im Fall einer attributweisen Präsentation von Information über Alternativen den Primacyoder den Recency-Effekt auslöst:

H1a: Wird der Vorteil von A zuerst und der Nachteil von A im Anschluss an nicht-diagnostische Information erst am Ende präsentiert, entsteht ein Präferenzvorteil für A (Primacy-Effekt durch Biased-Hypothesis-Testing).

H1b: Wird der Vorteil von A zuerst und der Nachteil von A im Anschluss an nicht-diagnostische Information erst am Ende präsentiert, entsteht ein Präferenznachteil für A (Recency-Effekt durch die Anwendung des in der Adaptationsniveautheorie postulierten Averaging-Prinzips).

\subsection{Mögliche Moderatoren}

Widersprüchliche Hypothesen legen die Frage nahe, ob es Bedingungen gibt, unter denen die eine oder die andere Hypothese zutrifft. Im Folgenden werden zwei mögliche Moderatoren diskutiert.

\subsubsection{Abbruch der Informationsverarbeitung ab einer bestimmten Menge an} aufgenommener Information

Mit zunehmender Menge an Informationseinheiten nimmt die Wahrscheinlichkeit zu, dass Personen nur einen Teil der Informationseinheiten verarbeiten. Wenn also z. B. in einer ersten Phase der Informationsübermittlung die Vorteile von A und die Nachteile von B vorgestellt werden und in einer zweiten Phase für sehr viele Merkmale nicht-diagnostische Information folgt, könnten die Personen ab einer bestimmten Menge an präsentierten Informationseinheiten die weitere Informationsverarbeitung abbrechen. Die in einer dritten Phase präsentierten Nachteile von A und Vorteile für B würden dann keine Rolle mehr dafür spielen, wofür sich die Personen entscheiden. Trifft diese These zu, wäre der in $H 1 a$ 
thematisierte Primacy-Effekt zu erwarten. Daraus resultiert die Frage, ab welcher Anzahl von Attributen deshalb der Primacy-Effekt zu erwarten ist, weil Personen die Verarbeitung von Information abbrechen.

Boyle/Saad (2011) führten ein Experiment durch, in dem sowohl Experten als auch Laien auf dem Gebiet von Digitalkameras zwei Digitalkameras A und B bewerten sollten. Die Probanden erhielten eine Liste, bestehend aus 25 Attributen. Die Personen konnten ein Merkmal auswählen und erhielten Information zu A und B. Dann konnten sie ein weiteres Merkmal auswählen, und sie bekamen wieder die Information zu A und B. Nach jedem Merkmal konnten sie jeweils entscheiden, ob sie Information für ein bisher noch nicht berücksichtigtes Attribut haben möchten oder den Informationsaufnahmeprozess beenden wollen und sich nun entscheiden. Die Autoren ermittelten, dass Experten im Mittel Information zu 13,33 Attributen und Laien im Mittel Information zu 11,05 Attributen abfragten, bevor sie den Informationsaufnahmeprozess beendeten. In einer ähnlichen Studie, die von Saad/Russo $(1996,261)$ durchgeführt wurde, mussten Personen 25-mal Entscheidungen zwischen jeweils zwei Appartements fällen. Die Autoren untersuchten an einer geringen Stichprobe von Probanden, wie viele Attribute die Personen verwendeten, bevor sie eine Entscheidung trafen. Diese Anzahl hing von den Probanden ab und lag zwischen 6,5 und 16,7 .

In den empirischen Studien, die hier präsentiert werden, soll ein Primacy-Effekt, der wegen einer Erschöpfung aufgrund einer hohen Menge an Information entsteht, vermieden werden. Daher wird die Anzahl der präsentierten Merkmale deutlich niedriger sein als von die von Saad/Russo (1996) und Boyle/Saad (2011) als „Erschöpfungsgrenze“ ermittelte Anzahl. Wir begrenzen die Anzahl der Attribute auf maximal sechs.

\subsubsection{Leicht oder schwer miteinander vergleichbare Merkmale}

Eine Antwort auf die Frage, unter welcher Bedingung es für Personen besonders einfach ist, Biased-Hypothesis-Testing vorzunehmen bzw. das in der Adaptationsniveautheorie unterstellte Averaging-Prinzip anzuwenden, könnte auch aus der Art der verglichenen Merkmale abgeleitet werden. Merkmale untereinander sind entweder schwer oder leicht zu vergleichen. Untereinander schwer vergleichbar sind technische Informationen (z. B. 120 kWh/Jahr Stromverbrauch mit 103 Liter Gebrauchsvolumen eines Kühlschranks) und technische Informationen mit Preisinformationen. Verhältnismässig leicht über Merkmale hinweg vergleichbar sind z. B. je Attribut existente Warentesturteile. Es ist schwierig, ein Urteil über zwei Notebooks A und B abzuleiten, wenn zuerst über die Optionen A und B informiert wird, wie viel sie in Kilogramm wiegen, und dann über A und B unterrichtet wird, wie hoch ihre Speicherkapazität in Megabyte ist. Die Information über Kilogramm und Megabyte lassen sich ohne hohen kognitiven Aufwand nicht einfach zusammenfassen, um A und B jeweils als Ganzes zu bewerten. Lägen die Bewertungen zu Gewicht und Speicherkapazität hingegen auf derselben Skala vor, wie dies im Fall von Warentesturteilen mit Noten zwischen 1 (sehr gut) und 5 (mangelhaft) üblich ist, können die attributweise präsentierten Informationen vergleichsweise leichter pro Alternative mittels des AveragingPrinzips aggregiert werden. Stone/Schkade (1994) bezeichnen Skalen wie die Warentestskala, anhand derer leicht über die Attribute hinweg verglichen werden kann, als „common scales".

Für die Anwendung von Biased-Hypothesis-Testing reicht pro Informationseinheit ordinale Information aus. Das heisst, pro Attribut müsste die Person nur wissen, ob A besser 
als B, gleich gut wie B oder schlechter als B ist. Für die Anwendung des in der Adaptationsniveautheorie postulierten Averaging-Prinzips müsste die Information ferner noch auf einer einheitlichen Skala metrisiert werden. Entweder liegt diese Information bereits in dieser Form vor oder sie müsste von den Personen erst vergleichbar gemacht werden. Im letzteren Fall müsste die Person z. B. Preisinformationen, technische Daten etc. in Nutzeneinheiten transformieren, was kognitiv sehr aufwändig ist. Liegen die Informationen über die Merkmale hinweg betrachtet auf derselben Skala vor, ist es vergleichsweise leichter, Urteile gemäss dem Averaging-Prinzip zu bilden. Stone/Schkade (1994, 264) behaupten, dass Personen dazu tendieren, weniger aufwendige Strategien zur Bewertung anzuwenden, wenn die Vergleiche über die Attribute hinweg schwierig sind. Demzufolge wird erwartet:

- Im Fall von schwer von Attribut zu Attribut vergleichbarer Information wenden Personen Biased-Hypothesis-Testing an. Dann wird ein Primacy-Effekt beobachtet.

- Im Fall von leicht von Attribut zu Attribut vergleichbarer Information wenden Personen sowohl Biased-Hypothesis-Testing als auch das das in der Adaptationsniveautheorie postulierte Averaging-Prinzip an. Ob insgesamt der Primacy-Effekt beobachtet werden kann, weil der mentale Prozess des Biased-Hypothesis-Testing überwiegt, ob insgesamt kein Effekt zu beobachten ist, weil beide Prozesse gleich intensiv sind, oder ob insgesamt der Recency-Effekt beobachtet werden kann, weil der mentale Prozess, der gemäss des Averaging-Prinzips zu erwarten ist, überwiegt, ist unklar.

Vergleicht man jedoch diese beiden Fälle, folgt:

H2: Werden anstelle von Informationen, die von Merkmal zu Merkmal schwer zu vergleichen sind, leicht von Merkmal zu Merkmal vergleichbare Informationen präsentiert, schwächt sich entweder die Stärke des beobachteten Primacy-Effekts ab oder anstelle eines Primacy-Effekts kann ein Recency-Effekt beobachtet werden.

Wenn der Averaging-Effekt (hergeleitet aus der Adaptationsniveautheorie) stärker wäre als der gegenteilige Effekt (hergeleitet aus der Theorie zum Biased-Hypothesis-Testing), tritt anstelle eines Primacy-Effekts ein Recency-Effekt auf.

Es ist anzumerken, dass in der Literatur auch diskutiert wird, ob verschiedene Ausprägungen ein und desselben Attributs schwer miteinander vergleichbar sein könnten. Man stelle sich beispielsweise zwei Nudel-Fertiggerichte vor, und ein Merkmal sei die Geschmacksrichtung (A: mit Lachs, B: mit Steinpilzen). Produktalternativen, die derartige Ausprägungen aufweisen, werden als „non-alignable assortment“ bezeichnet (Gourville/ Soman 2005, 385). In der hier vorliegenden Studie bedeutet „schwer vergleichbar“ hingegen, dass die Merkmale untereinander schwer vergleichbar sind, z. B. der Kaloriengehalt des Nudel-Fertiggerichts in kcal und sein Preis in €, was es erschwert, ein Gesamturteil pro Produkt zu bilden; innerhalb des jeweiligen Attributs sind die Ausprägungen der Alternativen naturgemäss sehr leicht vergleichbar.

\section{Experimente}

Im Folgenden werden die Befunde aus drei Experimenten dargestellt, in denen die Wirkung einer Variation der Position von Vor- und Nachteilen von Produktalternativen in einer attributweisen Informationspräsentation untersucht wurde. Im ersten Experiment wurde für schwer miteinander zu vergleichende Merkmale analysiert, ob ein Primacy- oder Recency-Effekt auftritt, wenn einer diagnostischen Information nicht-diagnostische Information folgt, bevor wieder diagnostische Information gegeben wird; damit kann eine Aus- 
sage über die Gültigkeit von $H 1 a$ oder $H 1 b$ getroffen werden. Erkenntnisziel des zweiten Experiments war die Antwort auf die Frage, ob das im ersten Experiment erzielte Ergebnis (entweder Primacy- oder Recency-Effekt) ebenfalls ermittelt werden kann, wenn der Preis als ein Merkmal hinzugenommen wird oder ob der Preis einen Sondereffekt erzeugt. Im dritten Experiment wurde schliesslich überprüft, ob die Stärke bzw. die Richtung des Reihenfolge-Effekts davon abhängen, dass die Merkmale untereinander entweder leicht oder schwer vergleichbar sind. Damit wird H2 überprüft. Die Experimente fanden zwischen 2009 und 2011 statt.

\subsection{Experiment 1}

Experimentelles Design: In der ersten Studie wurden zum einen die Reihenfolge von attributweise präsentierter, diagnostischer Information und zum anderen das Vorhandensein und die Menge an nicht-diagnostischer Information, die zwischen der diagnostischen Information enthalten ist, manipuliert. Das Experiment besitzt ein 2 (Reihenfolge der diagnostischen Information) $\times 3$ (Vorhandensein und Menge der nicht-diagnostischen Information) $\times 3$ (Produktkategorien) - Zwischengruppendesign. Der erste Faktor variiert in zwei Stufen, ob entweder zuerst über Merkmal 1 (Vorteil von Option A und Nachteil von Option B) und zuletzt über Merkmal 2 (Nachteil von A und Vorteil von B) informiert wird oder ob diese Sequenz umgedreht ist. In einem Pretest war zuvor erfolgreich überprüft worden, dass die für die Produktkategorien festgelegten Produktvorteile bzw. -nachteile auch in dieser Art und Weise von Testteilnehmern bewertet werden. Der zweite Faktor variiert in drei Stufen, ob und wie viel nicht-diagnostische Information zwischen der Präsentation der Information über die Merkmale 1 und 2 gegeben wird. Entweder wird die sequentielle Präsentation der Merkmale 1 und 2 nicht von nicht-diagnostischer Information unterbrochen, oder es wird nicht-diagnostische Information über zwei bzw. über vier Merkmale eingebunden. Aus der Kombination der beiden Faktoren resultieren sechs Experimentalbedingungen, die sich hinsichtlich der in einer Reihenfolge präsentierten Informationseinheiten unterscheiden. Der dritte Faktor war die Produktkategorie; das Experiment wurde am Beispiel von Wahlmöglichkeiten aus den Kategorien der elektrischen Zahnbürsten, Staubsauger und Fahrräder durchgeführt. Um das experimentelle Design zu verdeutlichen, wird am Beispiel der elektrischen Zahnbürsten in Tabelle 2 erklärt, wie die Informationspräsentation in den sechs Experimentalbedingungen ablief. Für die beiden weiteren Produktkategorien, Staubsauger und Fahrräder, existieren jeweils dieselben sechs Experimentalbedingungen; hier wurden lediglich die Inhalte der Merkmale ausgetauscht, um Staubsauger bzw. Fahrräder adäquat beschreiben zu können.

Procedere: Die Datenerhebung erfolgte an Computerbildschirmen. Die Teilnehmer des Experiments sahen eine Power-Point-Präsentation, die zwei Folien (im Fall der Reihenfolgen 1 und 2), vier Folien (im Fall der Reihenfolgen 3 und 4) oder sechs Folien (im Fall der Reihenfolgen 5 und 6) enthielt. Die jeweils nacheinander präsentierten Folien zeigten die Information, wie sie für das Beispiel Zahnbürsten in Tabelle 2 aufgeführt ist. Jede dieser Folien beschrieb beide Alternativen, also z. B. Zahnbürste A immer auf der linken und Zahnbürste B immer auf der rechten Seite der Folien in Form von Abbildungen der beiden Produkte unter Verwendung fiktiver Markennamen. Am Ende der jeweiligen Präsentation mussten die Probanden die bevorzugte Alternative unter der Annahme, beide würden gleich viel kosten, benennen. 


\begin{tabular}{|c|c|c|c|}
\hline \multirow{2}{*}{$\begin{array}{l}\text { Diagnostische } \\
\text { Information }\end{array}$} & \multicolumn{3}{|c|}{ Nicht-diagnostische Information } \\
\hline & keine & $\begin{array}{l}\text { wenig } \\
\text { (zwei Merkmale) }\end{array}$ & $\begin{array}{l}\text { viel } \\
\text { (vier Merkmale) }\end{array}$ \\
\hline $\begin{array}{l}\text { Zuerst Infor- } \\
\text { mation über } \\
\text { den Vorteil } \\
\text { von Option A } \\
\text { und zuletzt } \\
\text { Information } \\
\text { über den } \\
\text { Nachteil von } \\
\text { Option A }\end{array}$ & $\begin{array}{l}\text { Reihenfolge 1: } \\
\text { 1. A leistet } 1500 \text {, } \\
\text { B leistet } 800 \\
\text { Umdrehungen } \\
\text { pro Minute } \\
\text { 2. A wiegt } 425 \mathrm{~g} \text {, } \\
\text { B wiegt } 290 \mathrm{~g}\end{array}$ & $\begin{array}{l}\text { Reihenfolge 3: } \\
\text { 1. A leistet } 1500, \mathrm{~B} \\
\text { leistet } 800 \text { Umdre- } \\
\text { hungen pro Minute } \\
\text { 2. A und B haben } 300 \\
\text { Minuten Akkulauf- } \\
\text { zeit } \\
\text { 3. A und B verfügen } \\
\text { über neun Bürste- } \\
\text { naufsätze } \\
\text { 4. A wiegt } 425 \mathrm{~g}, \mathrm{~B} \\
\text { wiegt } 290 \mathrm{~g}\end{array}$ & $\begin{array}{l}\text { Reihenfolge 5: } \\
\text { 1. A leistet } 1500, \text { B leistet } 800 \\
\text { Umdrehungen pro Minute. } \\
\text { 2. A und B haben } 300 \text { Minuten } \\
\text { Akkulaufzeit } \\
\text { 3. A und B verfügen über neun } \\
\text { Bürstenaufsätze } \\
\text { 4. A und B besitzen ein Display } \\
\text { mit } 340 \times 240 \text { Pixel Auflö- } \\
\text { sung } \\
\text { 5. A und B haben } 20 \text { Monate } \\
\text { Garantie } \\
\text { 6. A wiegt } 425 \mathrm{~g}, \mathrm{~B} \text { wiegt } 290 \mathrm{~g}\end{array}$ \\
\hline $\begin{array}{l}\text { Zuerst Infor- } \\
\text { mation über } \\
\text { den Nachteil } \\
\text { von Option A } \\
\text { und zuletzt } \\
\text { Information } \\
\text { über den Vor- } \\
\text { teil von Opti- } \\
\text { on A }\end{array}$ & $\begin{array}{l}\text { Reihenfolge } 2: \\
\text { 1. A wiegt } 425 \mathrm{~g} \text {, } \\
\text { B wiegt } 290 \mathrm{~g} \\
\text { 2. A leistet } 1500 \text {, } \\
\text { B leistet } 800 \\
\text { Umdrehungen } \\
\text { pro Minute }\end{array}$ & $\begin{array}{l}\text { Reihenfolge } 4 \text { : } \\
\text { 1. A wiegt } 425 \mathrm{~g}, \mathrm{~B} \\
\text { wiegt } 290 \mathrm{~g} \\
\text { 2. A und B haben } 300 \\
\text { Minuten Akkulauf- } \\
\text { zeit } \\
\text { 3. A und B verfügen } \\
\text { über neun Bürste- } \\
\text { naufsätze } \\
\text { 4. A leistet } 1500, \mathrm{~B} \\
\text { leistet } 800 \text { Umdre- } \\
\text { hungen pro Minute }\end{array}$ & $\begin{array}{l}\text { Reihenfolge 6: } \\
\text { 1. A wiegt } 425 \mathrm{~g}, \mathrm{~B} \text { wiegt } 290 \mathrm{~g} \\
\text { 2. A und B haben } 300 \text { Minuten } \\
\text { Akkulaufzeit } \\
\text { 3. A und B verfügen über neun } \\
\text { Bürstenaufsätze } \\
\text { 4. A und B besitzen ein Display } \\
\text { mit } 340 \times 240 \text { Pixel Auflö- } \\
\text { sung } \\
\text { 5. A und B haben } 20 \text { Monate } \\
\text { Garantie } \\
\text { 6. A leistet } 1500 \text {, B leistet } 800 \\
\text { Umdrehungen pro Minute }\end{array}$ \\
\hline
\end{tabular}

Die Nummerierung 1., 2. etc. gibt an, in welcher Reihenfolge die Information präsentiert wurde.

Tabelle 2: Illustration des Experiment 1 zugrunde liegenden Design an einem Beispiel

Stichprobe: An diesem Experiment nahmen 720 Studenten teil. Auf jede Experimentalgruppe (sechs Reihenfolgen, drei Produktkategorien) entfielen ca. 40 Probanden. Die Personen waren zwischen 18 und 37 Jahre alt. 53,4\% der Testteilnehmer waren weiblich. Die Gruppen unterschieden sich nicht signifikant hinsichtlich der Verteilung des Alters oder des Geschlechts.

Ergebnis: Tabelle 3 zeigt, wie häufig Alternative A bzw. B bevorzugt wurden. Die in Prozent angegebenen Wahlanteile sind mit $p_{\mathrm{A}}$ und $p_{\mathrm{B}}$ bezeichnet. Sie addieren sich pro Experimentalbedingung (Reihenfolgen 1 bis 6) jeweils zu $100 \%$ auf.

Die Studie liefert den Befund, dass die Reihenfolge der Präsentation der diagnostischen Information ohne Einbindung von nicht-diagnostischer Information keinen Effekt auf die Wahlanteile hatte. Vergleicht man die Ergebnisse, die sich bei Reihenfolge 1 ergaben, mit denen, die im Fall der Reihenfolge 2 resultierten, so wird ersichtlich, dass sich der Wahlanteil von A - aggregiert über die drei Produktkategorien - auf 40,8\% (Reihenfolge 1) bzw. 38,3\% (Reihenfolge 2) beläuft. Diese Anteile unterscheiden sich nicht signifikant $\left(\chi^{2}(1)=0,157, p>0,60\right)$. Carlson et al. (2006) hatten in ihrem Experiment ebenfalls zwi- 


\begin{tabular}{|c|c|c|c|c|c|c|c|c|c|c|c|c|}
\hline \multirow[t]{3}{*}{$\begin{array}{l}\text { Produkt- } \\
\text { kategorie }\end{array}$} & \multicolumn{4}{|c|}{$\begin{array}{l}\text { Keine nicht-diagnostische } \\
\text { Information als } \\
\text { Unterbrechung }\end{array}$} & \multicolumn{4}{|c|}{$\begin{array}{l}\text { Zwei nicht-diagnostische } \\
\text { Informationen als } \\
\text { Unterbrechung }\end{array}$} & \multicolumn{4}{|c|}{$\begin{array}{l}\text { Vier nicht-diagnostische } \\
\text { Informationen als } \\
\text { Unterbrechung }\end{array}$} \\
\hline & \multicolumn{2}{|c|}{$\begin{array}{c}\text { Reihenfolge } \\
1\end{array}$} & \multicolumn{2}{|c|}{$\begin{array}{c}\text { Reihenfolge } \\
2\end{array}$} & \multicolumn{2}{|c|}{$\begin{array}{c}\text { Reihenfolge } \\
3\end{array}$} & \multicolumn{2}{|c|}{$\underset{4}{\text { Reihenfolge }}$} & \multicolumn{2}{|c|}{$\begin{array}{c}\text { Reihenfolge } \\
5\end{array}$} & \multicolumn{2}{|c|}{$\begin{array}{c}\text { Reihenfolge } \\
6\end{array}$} \\
\hline & $p_{\mathrm{A}}$ & $p_{\mathrm{B}}$ & $p_{\mathrm{A}}$ & $p_{\mathrm{B}}$ & $p_{\mathrm{A}}$ & $p_{\mathrm{B}}$ & $p_{\mathrm{A}}$ & $p_{\mathrm{B}}$ & $p_{\mathrm{A}}$ & $p_{\mathrm{B}}$ & $p_{\mathrm{A}}$ & $p_{\mathrm{B}}$ \\
\hline Fahrräder & 40,0 & 60,0 & 37,5 & 62,5 & 52,5 & 47,5 & 37,5 & 62,5 & 67,5 & 32,5 & 30,0 & 70,0 \\
\hline $\begin{array}{l}\text { Staubsau- } \\
\text { ger }\end{array}$ & 40,0 & 60,0 & 42,5 & 57,5 & 55,0 & 45,0 & 42,5 & 57,5 & 57,5 & 42,5 & 47,5 & 52,5 \\
\hline $\begin{array}{l}\text { Zahn- } \\
\text { bürsten }\end{array}$ & 42,5 & 57,5 & 35,0 & 65,0 & 65,0 & 35,0 & 27,5 & 72,5 & 70,0 & 30,0 & 30,0 & 70,0 \\
\hline Gesamt & 40,8 & 59,2 & 38,3 & 61,7 & 57,5 & 42,7 & 35,8 & 64,2 & 65,0 & 35,0 & 35,8 & 64,2 \\
\hline
\end{tabular}

Reihenfolge 1: 1. $x_{1 \mathrm{~A}}>x_{1 \mathrm{~B}} \rightarrow 2 . x_{2 \mathrm{~A}}<x_{2 \mathrm{~B}}$

Reihenfolge 2: 1. $x_{2 \mathrm{~A}}<x_{2 \mathrm{~B}} \rightarrow 2 . x_{1 \mathrm{~A}}>x_{1 \mathrm{~B}}$

Reihenfolge 3: 1. $x_{1 \mathrm{~A}}>x_{1 \mathrm{~B}} \rightarrow$ 2. $x_{3 \mathrm{~A}}=x_{3 \mathrm{~B}} \rightarrow$ 3. $x_{4 \mathrm{~A}}=x_{4 \mathrm{~B}} \rightarrow$ 4. $x_{2 \mathrm{~A}}<x_{2 \mathrm{~B}}$

Reihenfolge 4: 1. $x_{2 \mathrm{~A}}<x_{2 \mathrm{~B}} \rightarrow 2 \cdot x_{3 \mathrm{~A}}=x_{3 \mathrm{~B}} \rightarrow 3 \cdot x_{4 \mathrm{~A}}=x_{4 \mathrm{~B}} \rightarrow$ 4. $x_{1 \mathrm{~A}}>x_{1 \mathrm{~B}}$

Reihenfolge 5: 1. $x_{1 \mathrm{~A}}>x_{1 \mathrm{~B}} \rightarrow 2 \cdot x_{3 \mathrm{~A}}=x_{3 \mathrm{~B}} \rightarrow 3 \cdot x_{4 \mathrm{~A}}=x_{4 \mathrm{~B}} \rightarrow$ 4. $x_{5 \mathrm{~A}}=x_{5 \mathrm{~B}} \rightarrow$ 5. $x_{6 \mathrm{~A}}=x_{6 \mathrm{~B}} \rightarrow$ 6. $x_{2 \mathrm{~A}}<x_{2 \mathrm{~B}}$

Reihenfolge 6: 1. $x_{2 \mathrm{~A}}<x_{2 \mathrm{~B}} \rightarrow$ 2. $x_{3 \mathrm{~A}}=x_{3 \mathrm{~B}} \rightarrow 3 \cdot x_{4 \mathrm{~A}}=x_{4 \mathrm{~B}} \rightarrow$ 4. $x_{5 \mathrm{~A}}=x_{5 \mathrm{~B}} \rightarrow 5 \cdot x_{6 \mathrm{~A}}=x_{6 \mathrm{~B}} \rightarrow 6 \cdot x_{1 \mathrm{~A}}>x_{1 \mathrm{~B}}$

Tabelle 3: Ergebnis von Experiment 1

schen den diagnostischen Informationen keine nicht-diagnostische Information eingebunden; auch sie hatten für diesen Fall keinen Reihenfolge-Effekt beobachten können. War die Präsentation der diagnostischen Information jedoch durch nicht-diagnostische Informationen unterbrochen, erlangte die Alternative A einen Präferenzvorteil gegenüber Alternative B lediglich aufgrund dieser Unterbrechung. Das heisst, die Alternative A, über die bereits zu Beginn der Präsentation ein Vorteil übermittelt wurde, konnte einen höheren Wahlanteil erreichen (im Fall von zwei nicht-diagnostischen Informationen: 57,5\% > $35,8 \%, \chi^{2}(1)=11,317, p<0,001$; im Fall von vier nicht-diagnostischen Informationen: $\left.65,0 \%>35,8 \%, \chi^{2}(1)=20,418, p<0,001\right)$. Die erzielten Ergebnisse stützen die Gültigkeit von H1a (Primacy-Effekt). Es sind keine Auffälligkeiten erkennbar, wonach der beschriebene Reihenfolge-Effekt von der untersuchten Produktkategorie abhängen würde.

In Experiment 2 wird untersucht, ob der Primacy-Effekt ebenfalls beobachtet werden kann, wenn die Position der Preisinformation in der Reihenfolge variiert wird.

\subsection{Experiment 2}

Experimentelles Design: In diesem Experiment wurden die Alternativen A und B anhand von sechs Merkmalen beschrieben, wovon zwei Merkmale diagnostisch und vier Merkmale nicht-diagnostisch sind. Das experimentelle Design ist ein 2 (Reihenfolge der diagnostischen Information) $\times 2$ (Art der diagnostischen Information: nur technische Attribute vs. technische Attribute und Preis) $\times 4$ (Produktkategorien) - Zwischengruppendesign. Der erste Faktor variiert ebenso wie in Experiment 1 den Tatbestand, ob am Anfang oder am Ende der Präsentation hinsichtlich eines Vorteils von Alternative A informiert wird. Ein hoher Preis wird als Nachteil interpretiert (auf das Thema Preisausstrahlung wird nachfolgend eingegangen). Der zweite Faktor manipuliert, ob die Vor- und Nachteile entweder nur aus Information über technische Attribute oder sowohl aus Information über technische Attribute als auch über den Preis bestehen. Der dritte Faktor ergibt sich aus dem Tat- 
bestand, dass vier Produktkategorien betrachtet werden (Mikrowellengeräte, Staubsauger, Winterreifen, Übernachtung in einem Hotel). Tabelle 4 stellt exemplarisch für die Produktkategorie Mikrowellengeräte dar, wie die Manipulation der Reihenfolge der präsentierten Information vonstatten ging.

\begin{tabular}{|c|c|c|}
\hline \multirow{2}{*}{$\begin{array}{l}\text { Diagnostische } \\
\text { Information }\end{array}$} & \multicolumn{2}{|l|}{ Art der diagnostischen Information } \\
\hline & Nur technische Information & Technische und Preisinformation \\
\hline $\begin{array}{l}\text { Zuerst Informa- } \\
\text { tion über den } \\
\text { Vorteil von A } \\
\text { und zuletzt In- } \\
\text { formation über } \\
\text { den Nachteil } \\
\text { von A }\end{array}$ & $\begin{array}{l}\text { Reihenfolge 1: } \\
\text { 1. Garraumvolumen von A: } 24 \text {, } \\
\text { von B: } 16 \text { Liter } \\
\text { 2. Leistung von A und B } 950 \text { Watt } \\
\text { (ohne Grill) } \\
\text { 3. A und B bieten Heissluft von } 30 \\
\text { bis } 210 \text { Grad } \\
\text { 4. Abmessung von A und B: } \\
30 \times 50 \times 50 \mathrm{~cm} \\
\text { 5. Grilleistung A und B: } \\
\text { 1000 Watt } \\
\text { 6. A hat } 3 \text {, B hat } 6 \text { Leistungsstufen }\end{array}$ & $\begin{array}{l}\text { Reihenfolge 3: } \\
\text { 1. Garraumvolumen von A: } 24 \text {, } \\
\text { von B: } 16 \text { Liter } \\
\text { 2. Leistung von A und B } 950 \text { Watt } \\
\text { (ohne Grill) } \\
\text { 3. A und B bieten Heissluft von } \\
30 \text { bis } 210 \text { Grad } \\
\text { 4. Abmessung von A und B: } \\
30 \times 50 \times 50 \mathrm{~cm} \\
\text { 5. Grilleistung A und B: } \\
1000 \text { Watt } \\
\text { 6. A kostet } € 59,99 \text {, } \\
\text { B kostet } € 49,99\end{array}$ \\
\hline
\end{tabular}

Zuerst Informa- Reihenfolge 2:

tion über den 1. A hat 3, B hat 6 Leistungsstufen

Nachteil von A und zuletzt Information über den Vorteil von A
2. Leistung von $A$ und $B$ 950 Watt (ohne Grill)

3. A und B bieten Heissluft von 30 bis $210 \mathrm{Grad}$

4. Abmessung von $\mathrm{A}$ und $\mathrm{B}$ : $30 \times 50 \times 50 \mathrm{~cm}$

5. Grillleistung A und B: 1000 Watt

6. Garraumvolumen von A: 24, von B: 16 Liter

\section{Reihenfolge 4:}

1. A kostet $€ 59,99$, B kostet $€ 49,99$

2. Leistung von A und B 950 Watt (ohne Grill)

3. A und B bieten Heissluft von 30 bis $210 \mathrm{Grad}$

4. Abmessung von $A$ und $B$ : $30 \times 50 \times 50 \mathrm{~cm}$

5. Grillleistung A und B: 1000 Watt

6. Garraumvolumen von A: 24, von B: 16 Liter

Die Nummerierung 1., 2. etc. gibt an, in welcher Reihenfolge die Information präsentiert wurde.

Tabelle 4: Illustration des Experiment 2 zugrunde liegenden Designs an einem Beispiel

Im Fall der drei weiteren Produktkategorien waren die Merkmale durch geeignet ausgewählte, andere Inhalte ersetzt worden. Zwischen den beiden diagnostischen Informationen wurde in diesem Experiment relativ viel nicht-diagnostische Information eingebunden; damit sollte verhindert werden, dass eine Preisinformation, die zu Beginn einer Informationspräsentation gegeben wird, von den Testpersonen dazu verwendet wird, um auf Qualitäten zu schliessen. Wenn unmittelbar auf die Preisinformation vier nicht-diagnostische Informationen folgen, können Konsumenten nicht erwarten, dass das teure Produkt generell eine höhere Qualität hat. Dieser Aspekt wurde auch in einem Pretest zu diesem Experiment erfolgreich überprüft, indem einige Probanden pro Schritt der Informationspräsentation danach gefragt wurden, ob sie erwarten, dass das teure Produkt gleichzeitig das bessere Produkt ist. 
Procedere und Stichprobe: Die Datenerhebung erfolgte wie in Experiment 1 geschildert. An dieser Studie nahmen 512 Studenten teil. Auf jede Experimentalgruppe (vier Reihenfolgen, vier Produktkategorien) entfielen ca. 32 Probanden. Die Personen waren zwischen 19 und 32 Jahre alt (im Mittel 25,22 Jahre). 48,7\% der Testteilnehmer waren weiblich. Es gab keine signifikanten Unterschiede der Experimentalgruppen hinsichtlich des Alters und Geschlechts.

Ergebnis: Tabelle 5 kann entnommen werden, wie häufig Alternative A bzw. B bevorzugt wurden. Die in Prozent angegebenen Wahlanteile sind wieder mit $p_{\mathrm{A}}$ und $p_{\mathrm{B}}$ bezeichnet; in der Summe ergeben sie jeweils $100 \%$.

\begin{tabular}{|c|c|c|c|c|c|c|c|c|}
\hline \multirow[t]{3}{*}{$\begin{array}{l}\text { Produkt- } \\
\text { kategorie }\end{array}$} & \multicolumn{4}{|c|}{$\begin{array}{l}\text { Variation der Position von } \\
\text { Vor- und Nachteilen bezüglich } \\
\text { Qualitätsmerkmalen }\end{array}$} & \multicolumn{4}{|c|}{$\begin{array}{l}\text { Variation der Position von Vor- und } \\
\text { Nachteilen bzgl. Qualität und Preis }\end{array}$} \\
\hline & \multicolumn{2}{|c|}{ Reihenfolge 1} & \multicolumn{2}{|c|}{ Reihenfolge 2} & \multicolumn{2}{|c|}{ Reihenfolge 3} & \multicolumn{2}{|c|}{ Reihenfolge 4} \\
\hline & $p_{\mathrm{A}}$ & $p_{\mathrm{B}}$ & $p_{\mathrm{A}}$ & $p_{\mathrm{B}}$ & $p_{\mathrm{A}}$ & $p_{\mathrm{B}}$ & $p_{\mathrm{A}}$ & $p_{\mathrm{B}}$ \\
\hline Hotels & 62,5 & 37,5 & 34,4 & 65,6 & 75,0 & 25,0 & 43,8 & 56,3 \\
\hline $\begin{array}{l}\text { Mikrowellen- } \\
\text { gerät }\end{array}$ & 71,9 & 28,1 & 34,4 & 65,6 & 62,5 & 37,5 & 50,0 & 50,0 \\
\hline Staubsauger & 65,6 & 34,4 & 56,3 & 43,8 & 84,4 & 15,6 & 59,4 & 40,6 \\
\hline Winterreifen & 62,5 & 37,5 & 34,4 & 65,6 & 81,3 & 18,8 & 50,0 & 50,0 \\
\hline Gesamt & 65,6 & 34,4 & 39,8 & 60,2 & 75,8 & 24,2 & 50,8 & 49,2 \\
\hline
\end{tabular}

Reihenfolge 1: 1. $x_{1 \mathrm{~A}}>x_{1 \mathrm{~B}} \rightarrow 2 \cdot x_{2 \mathrm{~A}}=x_{2 \mathrm{~B}} \rightarrow 3 \cdot x_{3 \mathrm{~A}}=x_{3 \mathrm{~B}} \rightarrow$ 4. $x_{4 \mathrm{~A}}=x_{4 \mathrm{~B}} \rightarrow 5 \cdot x_{5 \mathrm{~A}}=x_{5 \mathrm{~B}} \rightarrow 6 \cdot x_{6 \mathrm{~A}}<x_{6 \mathrm{~B}}$ Reihenfolge 2: 1. $x_{6 \mathrm{~A}}<x_{6 \mathrm{~B}} \rightarrow 2 . x_{2 \mathrm{~A}}=x_{2 \mathrm{~B}} \rightarrow 3 \cdot x_{3 \mathrm{~A}}=x_{3 \mathrm{~B}} \rightarrow$ 4. $x_{4 \mathrm{~A}}=x_{4 \mathrm{~B}} \rightarrow 5 \cdot x_{5 \mathrm{~A}}=x_{5 \mathrm{~B}} \rightarrow 6 \cdot x_{1 \mathrm{~A}}>x_{1 \mathrm{~B}}$ Reihenfolge 3: 1. $x_{1 \mathrm{~A}}>x_{1 \mathrm{~B}} \rightarrow 2 . x_{2 \mathrm{~A}}=x_{2 \mathrm{~B}} \rightarrow 3 . x_{3 \mathrm{~A}}=x_{3 \mathrm{~B}} \rightarrow$ 4. $x_{4 \mathrm{~A}}=x_{4 \mathrm{~B}} \rightarrow 5 . x_{5 \mathrm{~A}}=x_{5 \mathrm{~B}} \rightarrow 6$. A teurer als B Reihenfolge 4: 1. A teurer als B $\rightarrow$ 2. $x_{2 \mathrm{~A}}=x_{2 \mathrm{~B}} \rightarrow 3 . x_{3 \mathrm{~A}}=x_{3 \mathrm{~B}} \rightarrow 4 . x_{4 \mathrm{~A}}=x_{4 \mathrm{~B}} \rightarrow 5 \cdot x_{5 \mathrm{~A}}=x_{5 \mathrm{~B}} \rightarrow 6 . x_{1 \mathrm{~A}}>x_{1 \mathrm{~B}}$

Tabelle 5: Ergebnis von Experiment 2

Wird die Position von Vor- und Nachteilen von Qualitätsmerkmalen variiert, resultiert der Primacy-Effekt, der schon in Experiment 1 beobachtet werden konnte. Der Wahlanteil von A sinkt - aggregiert über die Produktkategorien - von 65,6\% auf 39,8\%, wenn das vorteilhafte Qualitätsmerkmal nicht bereits an erster Stelle und das nachteilige Qualitätsmerkmal an letzter Stelle genannt werden (Reihenfolge 1), sondern diese Positionen vertauscht werden (Reihenfolge 2). Die Wahlanteile unterscheiden sich überzufällig (Vergleich der Reihenfolgen 1 und 2: $\left.\chi^{2}(1)=17,067, p<0,001\right)$. Wird eine Qualitätsinformation durch die Preisinformation ersetzt, tritt ebenfalls der Primacy-Effekt auf. Der Wahlanteil von A sinkt von 75,8\% auf 50,8\%. Auch dieser Rückgang ist signifikant (Vergleich der Reihenfolgen 3 und 4: $\left.\chi^{2}(1)=17,215, p<0,001\right)$. Die Preisinformation bereits an erster Stelle und sodann nicht-diagnostische Information zu präsentieren, ist also von Nachteil für den Wahlanteil des teuren Produkts und von Vorteil für den Wahlanteil des billigen Produkts. Die Ergebnisse sind über die Produktkategorien hinweg betrachtet stabil.

Die Experimente 1 und 2 deckten einen Primacy-Effekt auf. Sie hatten gezeigt, dass eine Wahlmöglichkeit einen Präferenzbonus erhält, wenn in einer attributweise ablaufenden Präsentation damit gestartet wird, den Vorteil dieser Option zu beschreiben, und wenn auf ihren Nachteil erst im Anschluss an den Einschub einiger nicht-diagnostischer Information 
hingewiesen wird. Diese Aussage kann auch dann getroffen werden, wenn der Preis als eine diagnostische Information in die Präsentation mit aufgenommen wird. In Experiment 3 wird abschliessend untersucht, ob sich der Primacy-Effekt abschwächt oder eventuell sogar der Recency-Effekt beobachtet wird, wenn anstelle schwer über die Merkmale hinweg vergleichbarer Informationen (wie in den Experimenten 1 und 2 getestet) leicht über die Merkmale hinweg vergleichbare Informationen vorliegen, um eine Entscheidung zu treffen.

\subsection{Experiment 3}

Experimentelles Design: Die Studie basierte auf einem 2 (Reihenfolge der diagnostischen Information) $\times 2$ (Art der diagnostischen Information: technische Daten vs. Warentesturteile) $\times 7$ (Produktkategorien) - Zwischengruppendesign. Die Alternativen sind anhand von vier diagnostischen Informationen und zwei nicht-diagnostischen Informationen beschrieben. Hinsichtlich der beiden letztgenannten Merkmale unterscheiden sich die Optionen nicht. In Faktor 1 wird variiert, ob zunächst attributweise anhand von zwei Merkmalen die Vorteile von A dargestellt und im Anschluss an zwei zusätzliche Merkmale, die nicht-diagnostisch sind, anhand von zwei weiteren Merkmalen die Nachteile von A präsentiert werden oder ob die Reihenfolge der Vor- und Nachteile vertauscht ist. Der zweite Faktor besteht in der Art der Information; hier werden technische Kenngrössen und Warentest-Noten unterschieden. Der dritte Faktor beinhaltet die untersuchten Produktkategorien (MP3-Player, Tintenstrahldrucker, Koffer, PC-Bildschirme, Sonnenbrillen, hochwertige Digitalkameras, geringwertige Digitalkameras). Tabelle 6 stellt für das Beispiel Tintenstrahldrucker dar, wie die Informationspräsentation in den Experimentalbedingungen ablief. Im Fall der Warentesturteile als Information lagen die Notendifferenzen pro diagnostisches Merkmal einheitlich bei 0,5 oder 0,6.

Pretest: In einem Pretest wurde überprüft, ob es Personen tatsächlich leichter fällt, Entscheidungen zu treffen, wenn Produktinformation attributweise in Form von Warentesturteilen anstelle in Form von technischen Daten präsentiert wird. Diese Prüfung fand nur am Beispiel eines der betrachteten Produkte (MP3-Player, Vergleich der Reihenfolgen 1 und 3) statt. 20 Probanden bekamen Information zu zwei MP3-Playern Information in der Art von Warentest-Urteilen, 20 weitere Probanden erhielten sie in Form der technischen Werte. Die Probanden entstammten derselben Grundgesamtheit wie die Personen, die an der Hauptstudie teilnahmen. Als abhängige Variable diente die Zustimmung zum Statement „Ich kann mich leicht entscheiden“ ( $1=$ trifft überhaupt nicht zu, $7=$ trifft voll und ganz zu). Die Entscheidungsleichtigkeit belief sich im Fall der Warentesturteile auf 4,6 und im Fall der technischen Information auf 2,8 $(t=4,12, p<0,001)$.

Procedere: In dieser Studie wurde den Testpersonen ebenfalls pro Attribut jeweils Information zu den Alternativen A und B präsentiert. Die beiden Alternativen wurden in einer Art Film erklärt. Dies heisst, es existierten sechs aufeinander folgende Filmabschnitte, die jeweils sechs Sekunden andauerten, in denen jeweils eines der sechs Attribute für beide Optionen vorgestellt wurde. Diese Filmabschnitte wurden dann so aneinandergefügt, dass die Reihenfolge der Merkmale gemäss dem experimentellen Design variierte. Die Datenerhebung erfolgte online. Die Testpersonen bekamen die Instruktion sich vorzustellen, dass beide Alternativen jeweils gleich viel kosten. Die Online-Erhebung erfolgte unter den Mitarbeitern in mehreren grossen Unternehmen. Die Mitarbeiter erhielten per Email einen Link auf die Befragung. Sie gelangten, indem sie diesen Link anklickten, auf eine Website, 
Diagnostische Art der diagnostischen Information

Information Technische Daten Warentesturteile

Zuerst Informa- Reihenfolge 1:

tion über den Vorteil von $\mathrm{Op}$ tion $\mathrm{A}$ und $\mathrm{zu}-$ letzt Information über den Nachteil von Option A Minute
1. A druckt 38, B druckt 31 Seiten/

2. Der Papierspeicher von A fasst 250 Blatt, der von B 120 Seiten

3. A und B haben einen USB2 Netzwerkanschluss

4. A und B können folgendes Papier bedrucken: Photo-, Glanz-, Normalpapier

5. Die Druckqualität von A ist $5760 \times 1440 \mathrm{dpi}$, die von B ist $9600 \times 2400 \mathrm{dpi}$

6. A bedruckt die Formate A4, A5, $20 \times 25,13 \times 18,10 \times 18,10 \times 15, \mathrm{~B}$ bedruckt ausserdem die Formate A6, $10 \times 25,9 \times 13,13 \times 20$
Zuerst Informa- Reihenfolge 2:

tion über den

Nachteil von

Option A und

zuletzt Informa-

tion über den

Vorteil von $\mathrm{Op}$ tion $\mathrm{A}$
1. Die Druckqualität von A ist $5760 \times 1440 \mathrm{dpi}$, die von B ist $9600 \times 2400 \mathrm{dpi}$

2. A bedruckt die Formate A4, A5, $20 \times 25,13 \times 18,10 \times 18,10 \times 15, \mathrm{~B}$ bedruckt ausserdem die Formate A6, $10 \times 25,9 \times 13,13 \times 20$

3. A und B haben einen USB2 Netzwerkanschluss

4. A und B können folgendes Papier bedrucken: Photo-, Glanz-, Normalpapier

5. A druckt 38, B druckt 31 Seiten/ Minute

6. Der Papierspeicher von A fasst 250 Blatt, der von B 120 Seiten

\section{Reihenfolge 3:}

1. Druckgeschwindigkeit A Note 1,5, B Note 2,1

2. Füllmenge des Papierspeichers A Note 2,1, B Note 2,7

3. Netzwerkanschlüsse A und B Note 1,4

4. Leistungsumfang hinsichtlich bedruckbaren Papiers A und B Note 2,1

5. Druckqualität A Note 2,5, B Note 2,0

6. Leistungsumfang hinsichtlich bedruckbarer Formate A Note 2,6, B Note 2,0

\section{Reihenfolge 4:}

1. Druckqualität A Note 2,5, B Note 2,0

2. Leistungsumfang hinsichtlich bedruckbarer Formate A Note 2,6, B Note 2,0

3. Netzwerkanschlüsse A und B Note 1,4

4. Leistungsumfang hinsichtlich bedruckbaren Papiers A und B Note 2,1

5. Druckgeschwindigkeit A Note 1,5 , B Note 2,1

6. Füllmenge des Papierspeichers A Note 2,1, B Note 2,7

Die Nummerierung 1., 2. etc. gibt an, in welcher Reihenfolge die Information präsentiert wurde.

Tabelle 6: Illustration des Experiment 3 zugrunde liegenden Designs an einem Beispiel

in der die Filme angesehen werden konnten und die Befragung technisch abgewickelt wurde.

Stichprobe: An diesem Experiment wirkten nur zu einem sehr geringen Anteil Studenten, möglicherweise als Werkstudenten, mit. Auf jede der 28 Testbedingungen (2 Reihenfolgen, 2 Arten von Merkmalsausprägungen, Alternativen aus 7 Produktkategorien) entfielen zwischen 36 und 48 Probanden. Insgesamt belief sich die Stichprobe auf 1.188 Personen. Diese Personen waren zwischen 18 und 56 Jahre alt. 48,1\% der Personen waren weiblich. Hinsichtlich des Alters und Geschlechts unterschieden sich die Experimentalgruppen nicht signifikant. 
Ergebnis: Die Befunde aus dieser Studie sind in Tabelle 7 enthalten. Sie enthält in Form von Prozentangaben, wie viele der Testteilnehmer sich für die Alternative A oder B entschieden hatten.

\begin{tabular}{|c|c|c|c|c|c|c|c|c|}
\hline \multirow[t]{3}{*}{ Produktkategorie } & \multicolumn{4}{|c|}{ Technische Daten } & \multicolumn{4}{|c|}{ Warentesturteile (Schulnoten) } \\
\hline & \multicolumn{2}{|c|}{$\begin{array}{c}\text { Reihenfolge } \\
1\end{array}$} & \multicolumn{2}{|c|}{$\begin{array}{c}\text { Reihenfolge } \\
2\end{array}$} & \multicolumn{2}{|c|}{$\begin{array}{c}\text { Reihenfolge } \\
3\end{array}$} & \multicolumn{2}{|c|}{$\begin{array}{c}\text { Reihenfolge } \\
4\end{array}$} \\
\hline & $p_{\mathrm{A}}$ & $p_{\mathrm{B}}$ & $\overline{p_{\mathrm{A}}}$ & $p_{\mathrm{B}}$ & $p_{\mathrm{A}}$ & $p_{\mathrm{B}}$ & $\begin{array}{l}p_{\mathrm{A}} \\
n_{1}\end{array}$ & $p_{\mathrm{B}}$ \\
\hline Drucker & 60,9 & 39,1 & 21,3 & 78,7 & 56,3 & 43,8 & 41,7 & 58,3 \\
\hline Geringwertige Kameras & 67,4 & 32,6 & 34,0 & 66,0 & 58,3 & 41,7 & 43,8 & 56,3 \\
\hline Hochwertige Kameras & 66,7 & 33,3 & 11,1 & 88,9 & 69,4 & 30,6 & 47,2 & 52,8 \\
\hline Koffer & 78,3 & 21,7 & 36,2 & 63,8 & 56,3 & 43,8 & 43,8 & 56,3 \\
\hline MP3-Player & 78,3 & 21,7 & 27,7 & 72,3 & 64,6 & 35,4 & 41,7 & 58,3 \\
\hline PC Bildschirm & 58,3 & 41,7 & 25.0 & 75,0 & 52,8 & 47,2 & 36,1 & 63,9 \\
\hline Sonnenbrillen & 63,9 & 36,1 & 22,2 & 77,8 & 58,3 & 41,7 & 41,7 & 58,3 \\
\hline Gesamt & 68,2 & 31,8 & 26,0 & 74,0 & 59,3 & 40,7 & 42,3 & 57,7 \\
\hline
\end{tabular}

Reihenfolgen 1 und 3: 1. $x_{1 \mathrm{~A}}>x_{1 \mathrm{~B}} \rightarrow 2 \cdot x_{2 \mathrm{~A}}>x_{2 \mathrm{~B}} \rightarrow 3 \cdot x_{3 \mathrm{~A}}=x_{3 \mathrm{~B}} \rightarrow 4 \cdot x_{4 \mathrm{~A}}=x_{4 \mathrm{~B}} \rightarrow 5 \cdot x_{5 \mathrm{~A}}<x_{5 \mathrm{~B}}$

$\rightarrow 6 . x_{6 \mathrm{~A}}<x_{6 \mathrm{~B}}$

Reihenfolgen 2 und 4: 1. $x_{5 \mathrm{~A}}<x_{5 \mathrm{~B}} \rightarrow$ 2. $x_{6 \mathrm{~A}}<x_{6 \mathrm{~B}} \rightarrow 3 \cdot x_{3 \mathrm{~A}}=x_{3 \mathrm{~B}} \rightarrow$ 4. $x_{4 \mathrm{~A}}=x_{4 \mathrm{~B}} \rightarrow 5 \cdot x_{1 \mathrm{~A}}>x_{1 \mathrm{~B}}$

$\rightarrow 6 . x_{2 \mathrm{~A}}>x_{2 \mathrm{~B}}$

Tabelle 7: Ergebnis von Experiment 3

Der Wahlanteil von Alternative A beläuft sich auf 68,2\%, wenn die Alternativen A und B durch technische Information beschrieben werden und die Vorteile von A eingangs und die Nachteile von A am Ende dargestellt werden. Der Wahlanteil von A ist nur 26,0\%, wenn die Nachteile von A eingangs und die Vorteile von A am Ende beschrieben werden. Die Reihenfolge der Merkmale, d. h. der Tatbestand, ob erst Vor- oder erst Nachteile gezeigt werden, hat also einen numerisch starken Einfluss auf den Ausgang der Entscheidung, d. h. es tritt ein starker Primacy-Effekt auf (Vergleich der Reihenfolgen 1 und 2: $\chi^{2}(1)=$ 104,790, $p<0,001)$. Ebenfalls ein Primacy-Effekt kann beobachtet werden, wenn die Alternativen attributweise durch Noten in der Art von Warentesturteilen beschrieben werden. Hier sinkt der Wahlanteil von A von 59,3\% auf 42,3\%, wenn die Reihenfolge, in der Vor- und Nachteile gezeigt werden, umgedreht wird (Vergleich der Reihenfolgen 3 und 4: $\left.\chi^{2}(1)=17,345, p<0,001\right)$. Damit wird nochmals $H 1 a$ für beide Arten von Merkmalen, d. h. sowohl für schwer als auch für leicht untereinander vergleichbare Merkmale, gestützt. Interessant für die Prüfung von $\mathrm{H} 2$ ist es, ob der Primacy-Effekt stärker ist, wenn anstelle von Warentesturteilen technische Merkmale verwendet werden, um die Alternativen sukzessive zu beschreiben. Da die Differenz zwischen 68,2\% - 26,0\% = 42,2\% grösser ist als die Differenz zwischen 59,3\% - 42,3\% = 17,0\% ( $z=4,593, p<0,001)$, wird H2 gestützt. Die Befunde sind wieder über die betrachteten Produktkategorien hinweg stabil.

\section{Zusammenfassung und Folgerungen}

\subsection{Zentrale Ergebnisse}

In den drei Experimenten konnte gezeigt werden, dass der Wahlanteil einer Alternative im Fall der attributweisen Informationspräsentation gesteigert werden kann, wenn zu Beginn 
der Vorteil dieser Option und im Anschluss nach Einbindung von nicht-diagnostischer Information ihr Nachteil dargestellt wird. Dies stellt einen Primacy-Effekt dar, den die Theorie des Biased-Hypothesis-Testing voraussagte. Dieser Effekt tritt sowohl bei Verwendung von schwer und als auch bei Verwendung von leicht über die Attribute hinweg vergleichbarer Information auf. Auch der Preis als Vor- oder Nachteil eines Produkts nahm hinsichtlich der Ergebnisse keine Sonderrolle ein, womit die Praktikerregel bestätigt wird, dass der Preis, sofern er den Produktnachteil darstellt, erst am Ende der Präsentation genannt werden sollte. Der beobachtete Primacy-Effekt fiel schwächer aus, wenn die Entscheidung auf Basis von Information, die über die Attribute hinweg leicht vergleichbar ist, gefällt wurde. Dies ist mit der Theorie konform, wonach neben dem durch Biased-Hypothesis-Testing erzeugten Primacy-Effekt simultan ein durch Averaging erzeugter RecencyEffekt entsteht und eine partielle Kompensation auftritt.

\subsection{Limitationen der Experimente}

In den Experimenten wurden jeweils zwei Alternativen vorgestellt, zwischen denen sich die Personen zu entscheiden hatten. Der Fall, dass mehr als zwei Wahlmöglichkeiten existieren, wurde nicht berücksichtigt, z. B. der Fall, dass drei Optionen A, B und C existieren. Es wurde nicht untersucht, ob ein Präferenzvorteil für Option A auch in diesem Fall entsteht, wenn ihre Vorteile gegenüber B und C am Anfang vorgestellt werden und dann nicht-diagnostische Information folgt. Des Weiteren bestanden die Informationseinheiten aus wenig komplexen Angaben. Die Wirkung längerer Texte anstelle von prägnanten technischen Daten und Preisen wurde hier nicht untersucht; im Verkaufsgespräch könnte sich ein Verkäufer jedoch ausführlich zu jedem Merkmal äussern. In diesem Fall könnte der Prozess des Biased-Hypothesis-Testing intensiver ablaufen und der Primacy-Effekt verstärkt werden. Schliesslich lag eine Laborsituation vor, in der sich die Testteilnehmer auf die Präsentation konzentrieren konnten. Die Frage, ob die beobachteten Effekte ebenfalls auftreten und ähnlich stark sind, wenn die Personen den Wahlmöglichkeiten nicht ihre volle Aufmerksamkeit zuteil werden lassen, kann diese Studie ebenfalls nicht beantworten.

\subsection{Beitrag für die Theorie und Ansatzpunkte für weitere Forschung}

Zunächst weist die Abhandlung auf eine weitere Möglichkeit hin, wie sich ein Reihenfolge-Effekt bewirken lässt. Informationseinheiten könnten attributweise präsentiert werden; wenn nicht-diagnostische Information eingebunden wird, entsteht ein Primacy-Effekt. Diese Art der Wirkung nicht-diagnostischer Information wurde in der Literatur bisher nicht betrachtet. Des Weiteren können der Studie Anhaltspunkte entnommen werden, welcher mentale Prozess überwiegt, wenn Personen sowohl Biased-Hypothesis-Testing durchführen als auch Averaging-Prozesse stattfinden könnten. Die hier vorgestellten Experimente zeigten, dass der Effekt des Biased-Hypothesis-Testing vergleichsweise stärker ist; ansonsten hätte anstelle des Primacy-Effekts ein Recency-Effekt beobachtet werden müssen.

Weitere Forschung könnte darauf abzielen, die im letzten Abschnitt skizzierten Limitationen der Experimente zu beseitigen. So wäre es wünschenswert, in zukünftiger Forschung die in Laborexperimenten erzielten Befunde durch Feldstudien zu validieren. Interessant wäre es auch, bei der Erforschung des Effekts der Position der Preisinformation nach der Preissensitivität der Konsumenten zu differenzieren. In weiterführender Forschung könnte auch untersucht werden, unter welchen Bedingungen Konsumenten eine attributweise In- 
formationspräsentation gegenüber anderen Formaten der Präsentation wie zum Beispiel in einer vollständigen Übersichtstabelle bevorzugen. Starke Konsumentenheterogenität hinsichtlich der Wichtigkeit verschiedener Attribute, Zeitdruck und der Wunsch von Personen, die in der betreffenden Kategorie wenig Erfahrung haben, im Rahmen des Entscheidungsprozesses geleitet zu werden, könnten Gegenstand derartiger Analysen sein.

\subsection{Empfehlungen für die Praxis}

Grundsätzlich kann der hier beschriebene Primacy-Effekt von einem Anbieter genutzt werden, wenn er zwei Wahlmöglichkeiten anbietet und daran interessiert ist, dem Kunden eine der Optionen besonders attraktiv erscheinen zu lassen. Dass Information attributweise präsentiert wird, kann man sich z. B. im Fall eines Verkaufsgesprächs zwischen Verkäufer und Kunde gut vorstellen - hier hat der Verkäufer die Möglichkeit, die Sequenz, in der Informationseinheiten übermittelt werden, zu steuern. Des Weiteren könnten Produkte eines Anbieters, die dieser auf einer Internet-Seite anbietet, in der beschriebenen Art und Weise dargestellt werden. Der Anbieter könnte neben den Produktabbildungen zuerst die Frage „Möchten Sie wissen, wie gut (die Produkte) hinsichtlich (Merkmals 1) sind? “ einblenden. Auf diese Weise informiert sich der Kunde über alle Optionen hinsichtlich eines ersten Merkmals. Nachdem dies geschehen ist, könnte der Anbieter dieselbe Frage zu Merkmal 2 einblenden, woraufhin der Kunde wieder neue Information abrufen kann. Im Wege der programmierten Sequenz der Merkmale, zu denen Information abgerufen kann, könnten die Entscheidungen des Kunden gesteuert werden. Der Effekt kann verstärkt werden, wenn die Merkmale untereinander schwer vergleichbar sind. Letztendlich kann diese Abhandlung die Phantasie der Programmierer von Online-Shops anregen, Produktinformation nicht nur alternativenweise zu zeigen: sie könnte auch attributweise präsentiert werden, wodurch einzelne Anbieter vom hier beschriebenen Primacy-Effekt profitieren können.

\section{Literaturhinweise}

Anderson, N.H. (1981): Foundations of Information Integration Theory, New York.

Asch, S.E. (1946): Forming Impressions of Personality, in: The Journal of Abnormal and Social Psychology, Jg. 41, Nr. 3, S. 258-290.

Bänsch, A. (2006): Verkaufspsychologie und Verkaufstechnik, 8. Auf., München.

Biswas, D./Biswas, A./Chatterjee, S. (2009): Making Judgments in a Two-Sequence Cue Environment: The Effects of Differential Cue Strengths, Order Sequence, and Distraction, in: Journal of Consumer Psychology, Jg. 19, Nr. 1, S. 88-97.

Biswas, D./Grewal, D./Roggeveen, A. (2010): How the Order of Sampled Experiential Products Affects Choice, in: Journal of Marketing Research, Jg. 47, Nr. 3, S. 508-519.

Bond, S.D./Carlson, K.A./Meloy, M.G./Russo, J.E./Tanner, R.J. (2007): Information Distortion in the Evaluation of a Single Option, in: Organizational Behavior and Human Decision Processes, Jg. 102, Nr. 2, S. 240-250.

Boyle, P.J./Saad, G. (2011): Product Expertise: A Moderator of Information Search in Sequential Choice, in: Marketing Management Journal, Jg. 21, Nr. 1, S. 84-96.

Bruine de Bruin, W./Keren, G. (2003): Order Effects in Sequentially Judged Options due to the Direction of Comparison, in: Organizational Behavior and Human Decision Processes, Jg. 92, Nr. 1-2, S. 91-101. 
Carlson, K.A./Pearo, L.K. (2004): Limiting Predecisional Distortion by Prior Valuation of Attribute Components, in: Organizational Behavior and Human Decision Processes, Jg. 94, Nr. 1, S. 48-59.

Carlson, K.A./Meloy, M.G./Lieb, D. (2009): Benefits Leader Reversion: How a Once-Preferred Product Recaptures its Standing, in: Journal of Marketing Research, Jg. 46, Nr. 6, S. 788-797.

Carlson, K.A./Meloy, M.G./Russo, J.E. (2006): Leader-Driven Primacy: Using Attribute Order to Affect Consumer Choice, in: Journal of Consumer Research, Jg. 32, Nr. 4, S. 513-518.

Darley, J.M./Gross, P.J. (1983): A Hypothesis-Confirming Bias in Labeling Effects, in: Journal of Personality and Social Psychology, Jg. 44, Nr. 1, S. 20-33.

Gourville, J.T./Soman, D. (2005): Overchoice and Assortment Type: When and Why Variety Backfires, in: Marketing Science, Jg. 24, Nr. 3, S. 382-395.

Gürhan-Canli, Z. (2003): The Effect of Expected Variability of Product Quality and Attribute Uniqueness on Family Brand Evaluations, in: Journal of Consumer Research, Jg. 30, Nr. 1, S. 105-114.

Ha, Y.-W./Hoch, S.J. (1989): Ambiguity, Processing Strategy, and Advertising-Evidence Interactions, in: Journal of Consumer Research, Jg. 16, Nr. 3, S. 354-360.

Haugtvedt, C.P./Wegener, D.T. (1994): Message Order Effects in Persuasion: An Attitude Strength Perspective, in: Journal of Consumer Research, Jg. 21, Nr. 1, S. 205-218.

Helson, H. (1947): Adaptation-Level as Frame of Reference for Prediction of Psychophysical Data, in: American Journal of Psychology, Jg. 60, Nr. 1, S. 1-29.

Helson, H. (1964): Adaptation-Level Theory: An Experimental and Systematic Approach to Behavior, New York.

Highhouse, S./Gallo, A. (1997): Order Effects in Personnel Decision Making, in: Human Performance, Jg. 10, Nr. 1, S. 31-45.

Hoch, S.J./Ha, Y.W. (1986): Consumer Learning Advertising and the Ambiguity of Product Experience, in: Journal of Consumer Research, Jg. 13, Nr. 2, S. 221-233.

Hogarth, R.M./Einhorn, H.J. (1992): Order Effects in Belief Updating: The Belief-Adjustment Model, in: Cognitive Psychology, Jg. 24, Nr. 1, S. 1-55.

Hovland, C.I./Mandel, W. (1957): Is There a "Law of Primacy" in Persuasion? in: Hovland, C.I. (Hrsg.): The Order of Presentation in Persuasion, New Haven, S. 13-22.

Igou, E.R./Bless, H. (2003): Inferring the Importance of Arguments: Order Effects and Conversational Rules, in: Journal of Experimental Social Psychology, Jg. 39, Nr. 1, S. 91-99.

Ikea (2012): Frag einfach Anna, http://askanna-de.ikea.com/ikea-de/cgi-bin/ikea-de.cgi (Abfragedatum 16.1.2012).

Kardes, F.R./Herr, P.M. (1990): Order Effects in Consumer Judgment, Choice and Memory: The Role of Initial Processing Goals, in: Goldberg, M.E./Gorn, G./Pollay, W. (Hrgs.): Advances in Consumer Research, Jg. 17, Provo, UT, S. 541-546.

Kjoer, T./Bech, M./Gyrd-Hansen, D./Hart-Hansen, K. (2006): Ordering Effect and Price Sensitivity in Discrete Choice Experiments: Need We Worry? in: Health Economics, Jg. 15, Nr. 11, S. $1217-1228$.

Kröner, B. (1974): Adaptationsniveau-Bildung in der Wahrnehmung und psychophysiologischen Aktivierung, Diss., Bochum.

Lana, R.E. (1961): Familiarity and the Order of Presentation of Persuasive Communications, in: Journal of Abnormal and Social Psychology, Jg. 62, Nr. 3, S. 573-577. 
Leesch, C./Herrmann, A./Landwehr, J.R. (2010): Auf den Rangplatz kommt es an - Wirkung der Rangfolge von Produkten auf das Entscheidungsverhalten, in: Zeitschrift für Betriebswirtschaft, Jg. 80, Nr. 4, S. 441-466.

Levav, J./Heitmann, M./Herrmann, A./Iyengar, S.S. (2010): Order in Product Customization Decisions: Evidence from Field Experiments, in: Journal of Political Economy, Jg. 118, Nr. 2, S. 274-299.

Li, Y./Epley, N. (2009): When the Best Appears to be Saved for Last: Serial Position Effects on Choice, in: Journal of Behavioral Decision Making, Jg. 22, Nr. 4, S. 378-389.

Lund, F.H. (1925): The Psychology of Belief, in: The Journal of Abnormal and Social Psychology, Jg. 20, Nr. 2, S. 174-196.

Malaviya, P./Sternthal, B. (2009): Parity Product Features Can Enhance or Dilute Brand Evaluation: The Influence of Goal Orientation and Presentation Format, in: Journal of Consumer Research, Jg. 36, Nr. 1, S. 112-121.

Meyvis, T./Janiszewski, C. (2002): Consumers' Beliefs about Product Benefits: The Effect of Obviously Irrelevant Product Information, in: Journal of Consumer Research, Jg. 28, Nr. 4, S. 618-635.

Nisbett, R.E./Zuckier, H./Lemley, R.E. (1981): The Dilution Effect: Nondiagnostic Information Weakens the Implication of Diagnostic Information, in: Cognitive Psychology, Jg. 13, Nr. 2, S. 248-277.

Petty, R.E./Cacioppo, J.T. (1986): Communication and Persuasion - Central and Peripheral Routes to Attitude Change, New York.

Petty, R.E./Cacioppo, J.T./Schumann, D. (1983): Central and Peripheral Routes to Advertising Effectiveness: The Moderating Role of Involvement, in: Journal of Consumer Research, Jg. 10, Nr. 2, S. 135-146.

Petty, R.E./Tormala, Z.L./Hawkins, C./Wegener, D.T. (2001): Motivation to Think and Order Effects in Persuasion: The Moderating Role of Chunking, in: Personality and Social Psychology Bulletin, Jg. 27, Nr. 3, S. 332-344.

Pieters, R.G./Bijmolt, T.H.A. (1997): Consumer Memory for Television Advertising: A Field Study of Duration, Serial Position, and Competition Effects, in: Journal of Consumer Research, Jg. 23, Nr. 4, S. 362-372.

Polman, E. (2010): Information Distortion in Self-Other Decision Making, in: Journal of Experimental Social Psychology, Jg. 46, Nr. 2, S. 432-435.

Posavac, S.S./Kardes, F.R./Brakus, J.J. (2010): Focused Induced Tunnel Vision in Managerial Judgement and Decision Making: The Peril and the Antidote, in: Organizational Behavior and Human Decision Processes, Jg. 113, Nr. 2, S. 102-111.

Raanaas, R.K./Magnussen, S. (2006): Serial Position Effects in Implicit Memory for Multiple-Digit Numbers, in: Psychological Research, Jg. 70, Nr. 1, S. 26-31.

Russo, J.E./Carlson, K.A./Meloy, M.G./Yong, K. (2008): The Goal of Consistency as a Cause of Information Distortion, in: Journal of Experimental Psychology, Jg. 137, Nr. 3, S. 456-470.

Russo, J.E./Chaxel, A.-S. (2010): How Persuasive Messages Can Influence Behavior without Awareness, in: Journal of Consumer Psychology, Jg. 20, Nr. 3, S. 338-342.

Russo, J.E./Medvec, V.H./Meloy, M.G. (1996): The Distortion of Information during Decisions, in: Organizational Behavior and Human Decision Processes, Jg. 66, Nr. 1, S. 102-110.

Russo, J.E./Meloy, M.G./Medvec, V.H. (1998): Predecisional Distortion of Product Information, in: Journal of Marketing Research, Jg. 35, Nr. 4, S. 438-452. 
Russo, J.E./Meloy, M.G./Wilks, T.J. (2000): Predecisonal Distortion of Information Auditors and Salespersons, in: Management Science, Jg. 46, Nr. 1, S. 13-27.

Saad, G./Russo, J.E. (1996): Stopping Criteria in Sequential Choice, in: Organizational Behavior and Human Decision Processes, Jg. 67, Nr. 3, S. 258-270.

Sanbonmatsu, D.M./Posavac, S.S./Stasney, R. (1997): The Subjective Beliefs Underlying Probability Overestimation, in: Journal of Experimental Social Psychology, Jg. 33, Nr. 3, S. 276-295.

Sarris, V./Lauterbach, W. (1980): Zur konzeptuellen Bedeutung von psychologischen Bezugssystemen: Wahrnehmungspsychologische Grundlagen, in: Lauterbach, W./Sarris, V. (Hrsg.): Beiträge zur psychologischen Bezugsystemforschung, Bern, S. 15-54.

Scarpi, D. (2004): Effects of Presentation Order on Product Evaluation: An Empirical Analysis, in: International Review of Retail, Distribution and Consumer Research, Jg. 14, Nr. 3, S. 309-319.

Sitmalidis, S. (1976): Helsons Adaptationsniveautheorie und Wittes Bezugssystemtheorie: Ein experimenteller Vergleich ihres Voraussagewerts, Meisenheim am Glan.

Snyder, M./Cantor, N. (1979): Testing Hypothesis about Other People: The Use of Historical Knowledge, in: Journal of Experimental Social Psychology, Jg. 15, Nr. 4, S. 340-342.

Snyder, M./Swann, W.B. (1978): Hypothesis Testing Processes in Social Interaction, in: Journal of Personality and Social Psychology, Jg. 36, Nr. 11, S. 1202-1212.

Stone, D.N./Schkade, D.A. (1994): Effects of Attribute Scales on Process and Performance in Multiattribute Choice, in: Organizational Behavior and Human Decision Processes, Jg. 59, Nr. 2, S. 261-287.

Tavassoli, N.T./Lee, Y.H. (2004): The Effect of Attribute Order on Judgement in Chinese and English, in: Journal of Experimental Psychology: Applied, Jg. 10, Nr. 4, S. 258-266.

Terry, W.S. (2005): Serial Position Effects in Recall of Television Commercials, in: The Journal of General Psychology, Jg. 132, Nr. 2, S. 151-163.

Troutman, M.C./Shanteau, J. (1977): Inferences Based on Nondiagnostic Information, in: Organizational Behavior and Human Performance, Jg. 19, Nr. 1, S. 43-55.

Zeit Online (2012): CHE Hochschulranking, http://ranking.zeit.de/che2011/de/ (Abfragedatum 16.1.2012).

Heribert Gierl, Dr., ist Professor für Betriebswirtschaftslehre mit dem Schwerpunkt Marketing an der Universität Augsburg.

Karin Stiegelmayr, Dr., ist Mitarbeiterin an diesem Lehrstuhl.

Anschrift: Universität Augsburg, Universitätsstr. 16, D-86159 Augsburg, Tel.: +49 (0)821/598-4052, Fax: +49 (0)821/598-4216, E-Mail: heribert.gierl@wiwi.uni-augsburg.de

Für die Mitarbeit an verschiedenen Pretests und an der Erhebung von Teilen der Datenbasis der hier vorgestellten Studien danken die Autoren Frau Tina Berti, Frau Marine Carou, Herrn Erol Haciahmet, Herrn Nico Reichelmann und Herrn Norbert Schwärzler. 